\section{TUNNELING FROM SUPER- TO NORMAL- DEFORMED MINIMA IN NUCLEI}

\author{
TENG LEK KHOO
}

Physics Division, Argonne National Laboratory, 9700 S. Cass Avenue Argonne, IL60439, USA

E-mail: khoo@anl.gov

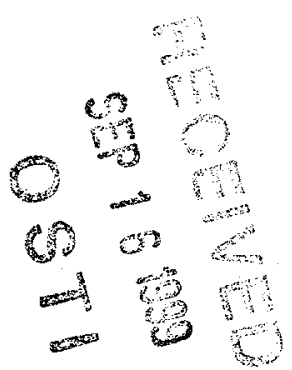

\begin{abstract}
An excited minimum, or false vacuum, gives rise to a highly elongated superdeformed (SD) nucleus. A brief review of superdeformation is given, with emphasis on the tunneling from the false to the true vacuum, which occurs in the feeding and decay of SD bands. During the feeding process the tunneling is between hot states, while in the decay it is from a cold to a hot state. The $\gamma$ spectra connecting SD and normal-deformed (ND) states provide information on several physics issues: the decay mechanism; the spin/parity quantum numbers, energies and microscopic structures of SD bands; the origin of identical SD bands; the quenching of pairing with excitation energy; and the chaoticity of excited ND states at 2.5 $5 \mathrm{MeV}$. Other examples of tunneling in nuclei, which are briefly described, include the possible role of tunneling in $\Delta \mathrm{I}=4$ bifurcation in SD bands, sub-barrier fusion and proton emitters.
\end{abstract}

\section{Introduction}

The study of superdeformed nuclei provides new insights into the structure of the nucleus. It also illustrates that the nucleus is a laboratory for investigating more general physics phenomena such as tunneling, chaos and phase transitions in mesoscopic systems. Relating different fields of physics and identifying the applications of common principles is not only interesting but also beneficial. On one hand, the nucleus can provide interesting data for the study of these general phenomena, e.g. for understanding tunneling in complex systems. On the other hand, new insights into the nucleus can also be obtained from this approach. Finally, perspectives and techniques can be profitably shared among the different areas of physics.

These lecture notes will provide a description of superdeformation in nuclei and will emphasize the role of tunneling from the superdeformed to normaldeformed potential wells. As an experimentalist, I shall highlight what has and can be measured, with the hope that this will provide some stimulation to theoreticians. 


\section{DISCLAIMER}

This report was prepared as an account of work sponsored by an agency of the United States Government. Neither the United States Government nor any agency thereof, nor any of their employees, make any warranty, express or implied, or assumes any legal liability or responsibility for the accuracy, completeness, or usefuiness of any information, apparatus, product, or process disclosed, or represents that its use would not infringe privately owned rights. Reference herein to any specific commercial product, process, or service by trade name, trademark, manufacturer, or otherwise does not necessarily constitute or imply its endorsement, recommendation, or favoring by the United States Government or any agency thereof. The views and opinions of authors expressed herein do not necessarily state or reflect those of the United States Government or any agency thereof. 


\section{DISCLAIMER}

Portions of this document may be illegible in electronic image products. Images are produced from the best available original document. 


\section{Superdeformation in nuclei}

\subsection{General Properties}

The nucleus, with a finite number of nucleons, is a mesoscopic object. The mesoscopic nature is illustrated by the fact that its energy can be given in terms of a superposition of a macroscopic liquid-drop term and a microscopic quantal shell-correction term. In a major advance in nuclear theory, Strutinsky ${ }^{1}$ developed the method to compute the shell-correction energy. The shell energy has a profound impact on nuclear behavior and leads to a number of striking consequences. One is the increased binding (by up to $7 \mathrm{MeV}$ ) in the heaviest elements, with atomic number larger than 104, resulting in the creation of a fission barrier, where classically none would exist at all. The existence of these elements is possible only because of the extra binding from the shell-correction energy. Hence, the production and discovery ${ }^{2}$ of the heaviest elements with $\mathrm{Z}$ up to 112 constitute some of the more exciting achievements in nuclear physics.

Another striking example (illustrated in Fig. 1) is the creation of a secondary minimum, or false vacuum, in the potential energy surface, at a de-

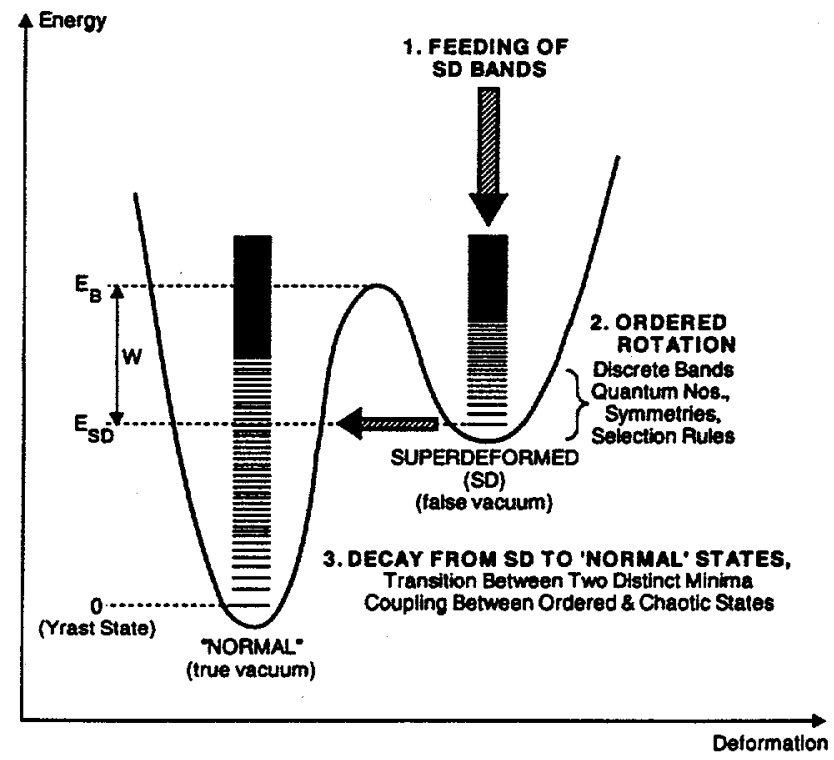

Figure 1: Sketch of potential energy vs. deformation, showing two minima created by the shell-correction energy. The three stages in the "life" of a superdeformed band are indicated, together with some characteristic properties. 
formation larger than the one corresponding to the lowest minimum (true vacuum). At the superdeformed minimum, the ratio of the long to short axes is around two. The first manifestation of a secondary minimum was the occurrence of fission isomers (reviewed in Ref. ${ }^{3}$ ). A later manifestation was the existence of superdeformed (SD) bands, which exhibit impressive series of equi- spaced (picket-fence) $\gamma$ transitions (reviewed in Refs. ${ }^{4,5}$ ). An example of a $\gamma$ spectrum from a SD band is shown in Fig. 2, taken from Ref. ${ }^{6}$.

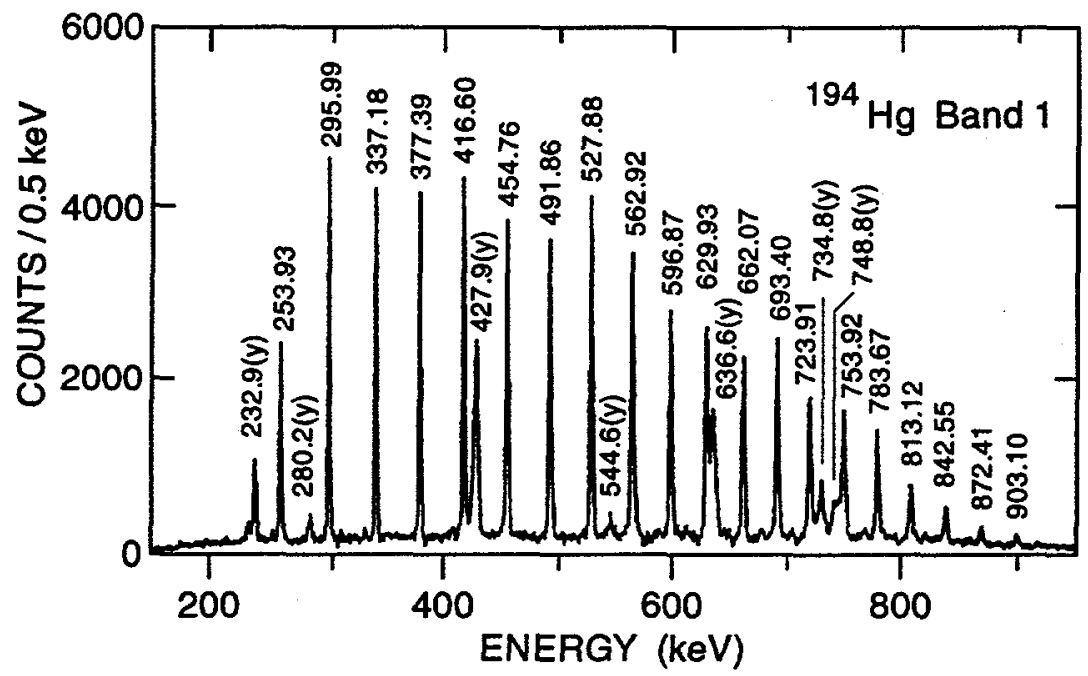

Figure 2: A typical spectrum ${ }^{6}$ of a SD band - from ${ }^{194} \mathrm{Hg}$ - showing a characteristic picket-fence spectrum with lines of nearly equal spacing. Transitions labeled (y) are normaldeformed yrast transitions, which are fed after decay from the SD band.

Although the lowest states in the SD false vacuum lie at high excitation energies above the ground or yrast states, they are isolated by a barrier separating SD and normal-deformed (ND) states. (A yrast state is defined as the lowest-lying level at each spin.) As a consequence, the lowest SD states are cold states, characterized by good quantum numbers and symmetries, and their decays are governed by selection rules (Fig. 1). In contrast, ND states that are at the same energies may be described as chaotic compound states, where quantum numbers (apart from spin and parity) are largely lost.

The occurrence of superdeformation owes it origin to large shell gaps. In a harmonic oscillator potential, energy gaps occur when the ratio of the longto short- axes is a rational ratio ${ }^{7}$, e.g. $2 / 1$, corresponding to closed classical orbits. The existence of a spin-orbit interaction in nuclei modifies the pure 
harmonic oscillator potential, but the occurrence of shell gaps at large deformation persists. When the proton and neutron numbers are both favorable for the occurrence of shell gaps, superdeformation is found. This leads to SD nuclei, which congregate in local regions in the chart of nuclides, with mass numbers around 80,130,150, 190 and 240 (Fig. 3, from Ref. ${ }^{8}$ ).

Fission Isomers

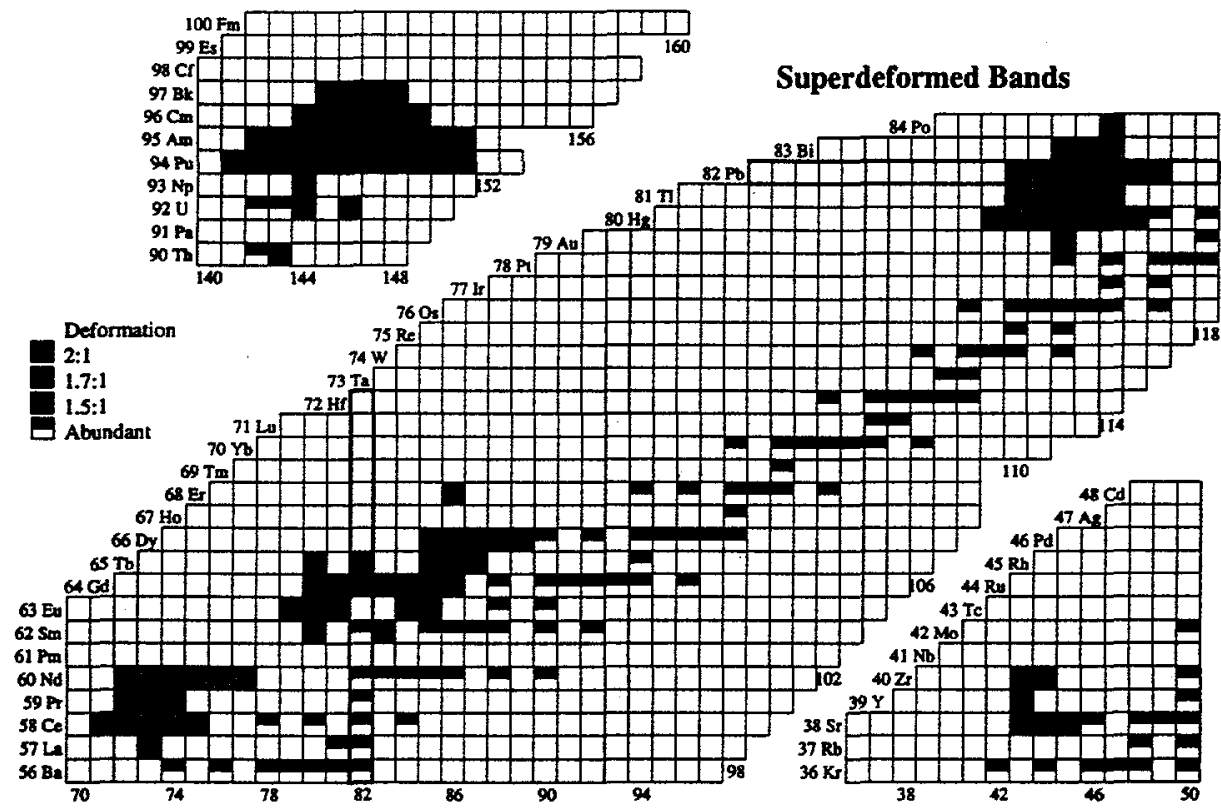

Figure 3: Chart of nuclides, showing local regions of superdeformation in the $A \sim 80,130$, 150,190 and 240 regions; from Ref. ${ }^{8}$.

A fascinating feature of almost all SD bands is the sudden drop in intraband transition intensity at low spin, after a string of up to 20 consecutive transitions (Fig. 4). In other words, after a long sequence of transitions within the false vacuum, there is a sudden decay to the true vacuum.

A $\gamma$ cascade which flows through a SD minimum has three stages (Fig. 1): (a) feeding and trapping into the SD well, (b) intraband transitions within SD bands, and (c) decay from SD to ND states. In stage (a), hot compound nuclear states cool via $\gamma$ emission. This stage involves the coupling of hot SD and ND states, which includes tunneling between hot states on either side of the barrier. A small fraction (typically around $1 \%$ ) of the cascades becomes trapped in the 


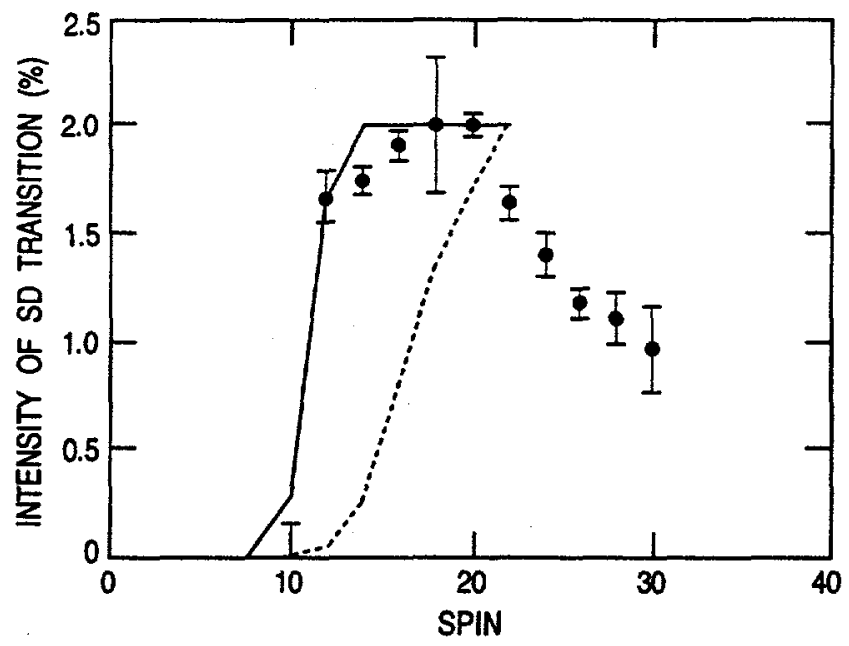

Figure 4: Intensities of SD-band transitions in ${ }^{192} \mathrm{Hg}$ as a function of initial spin, showing a sudden drop around spin 12. The solid and dashed lines are results of calculations using the Vigezxi model ${ }^{20}$, with the SD well depth W(I) assumed to either increase with spin (solid line) or remain constant (dashed line).

SD minimum, leading to stage (b), where equally-spaced transitions connect cold SD-band members. Here, selection rules imposed by quantum numbers govern the $\gamma$ decay, confining the decay to states of the same $K$ (projection of total angular momentum on the symmetry axis), i.e. within a band. With decreasing spin, the excitation energy of the trapped SD state above the ND yrast line rises. It then becomes embedded in a sea of ND states with increasing level density. Finally, when the SD state becomes unavoidably close to a ND level, a sudden tunneling from the cold SD state to the hot ND states occurs - stage (c).

\subsection{Tunneling from SD States}

Several interesting aspects of the tunneling process are worth noting. The initial SD state is a cold ordered state, characterized by good quantum numbers, whereas the final hot states are (probably) not. At high excitation energy, say near the neutron separation energy, hot ND states are described as chaotic states $^{8}$. The hot ND states (with $\sim 4 \mathrm{MeV}$ excitation energies) to which the SD states tunnel appear to be chaotic (although it still remains to be established whether full chaos is reached). 
By analogy with a classical system, where chaos implies no constants of motion, in a quantum system chaos implies a loss of certain quantum numbers. At high excitation energy the residual nucleon interactions mix states over an energy interval. When the spreading width $\Gamma_{Q}$ associated with a quantum number $Q$ becomes much larger than the average level spacing $D$, i.e. $\Gamma_{Q} / D \gg 1$, chaos is attained. ${ }^{10} \mathrm{An}$ interesting question is how the good quantum numbers dissolve in the tunneling process? One might also ask if the formalism developed for chaos-assisted tunneling ${ }^{11}$ could be usefully applied to this nuclear problem, or whether tunneling from SD states can provide an interesting example of chaos- assisted tunneling. Finally, it should be noted that the tunneling occurs in a multidimensional space, as the nuclear shape is characterized by at least two parameters, $\beta$ and $\gamma$ (which are defined later).

\subsection{Experiments}
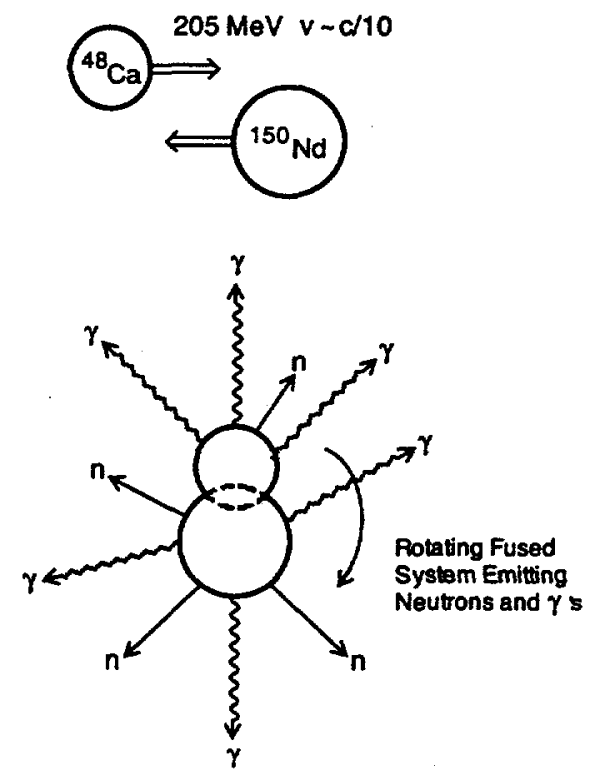

Figure 5: Cartoon of two nuclei fusing to form the compound nucleus198Hg, followed by emission of 4 neutrons and 20- $25 \gamma$ rays.

Rapidly spinning nuclei are usually formed in heavy-ion fusion reactions, where a heavy-ion projectile fuses with a target nucleus (Fig. 5). The hot, rapidly-rotating, compound nucleus cools by evaporating neutrons, then $\gamma$ rays. 
Most of what we have learned about superdeformed bands has been deduced from the $\gamma$ rays. A rapid growth of knowledge has occurred with the operation of large, powerful $\gamma$-ray detector arrays of Compton-suppressed Ge detectors, such as Gammasphere ${ }^{12}$ (which has been located in Berkeley and is now in Argonne) or Euroball (which is now in Legnaro). In fact, superdeformation provided one of the original motivations for constructing these arrays. Fig. 6 shows a photograph of Gammasphere.

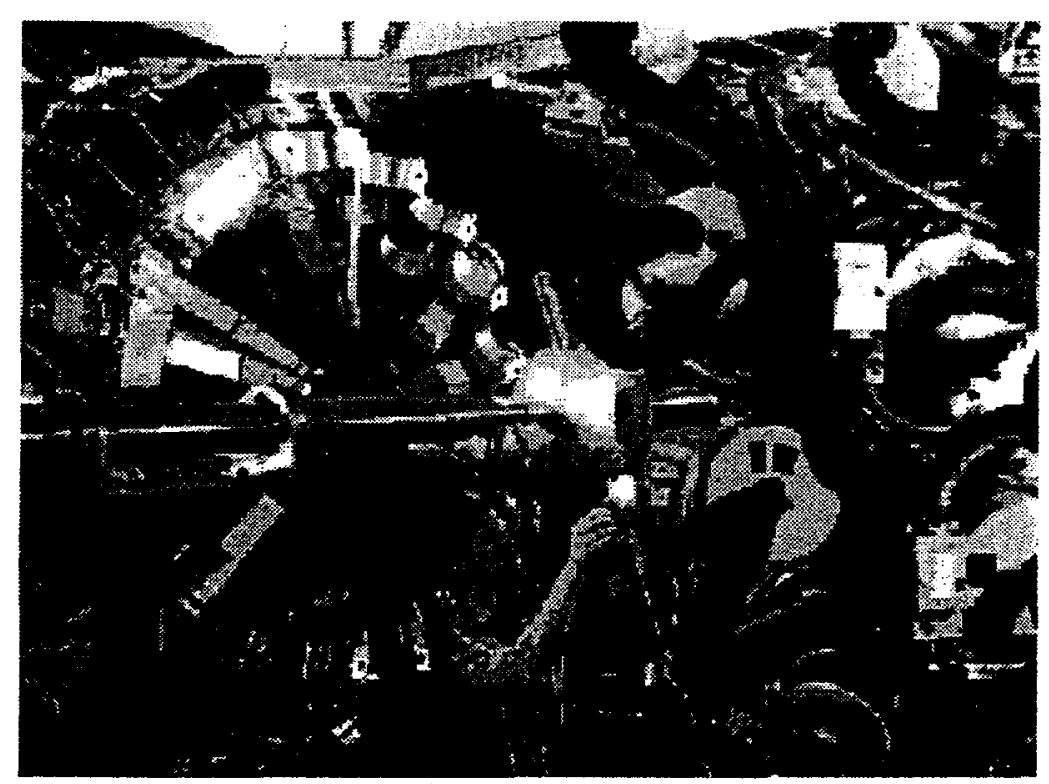

Figure 6: Photograph of Gammasphere ${ }^{12}$.

The shape of a hot compound nucleus is often not well defined because thermal fluctuations, present in a mesoscopic system, lead to a superposition of many shapes. However, as the nucleus cools towards the yrast line, a variety of ND and SD shapes become defined. These are illustrated in Fig. 7, which also shows the $\beta-\gamma$ values characterizing these shapes. Nuclear shapes are usually described by two deformation parameters: $\beta$, a measure of elongation, and $\gamma$, a measure of non-axiality. Typically, a SD shape has $\beta \sim 0.5, \gamma \sim 0$, while hot ND states have a considerable spread in $\beta$ and $\gamma$ because of thermal shape fluctuations. 

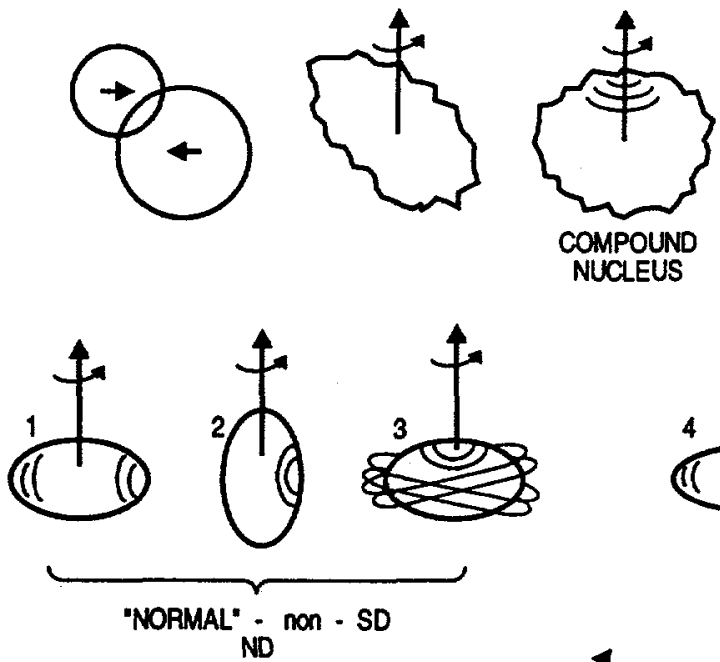

ND

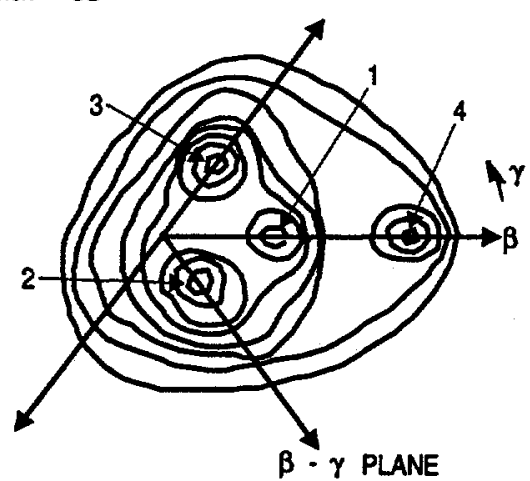

Figure 7: Sequence of nuclear shapes formed after fusion. The shapes in the hot excited phase are largely undefined because of thermal fluctuations, which are present due to the mesoscopic nature of the nucleus. Upon cooling to the vicinity of the yrast line, a variety of defined shapes (labeled 1-4) may be encountered, which are described in terms of the indicated $\beta$ - and $\gamma$ - shape parameters.

\subsection{Feeding of SD bands}

The population of SD bands can be understood in terms of a statistical process (i.e. one governed by level densities), provided tunneling between hot SD and ND states is taken into account. ${ }^{13,14}$ As the nucleus cools by emission of 
so-called statistical $\gamma$ rays, a small fraction $(\sim 1 \%)$ are trapped within the SD minimum. Once they are in the "ground" state in this false vacuum, no further cooling is possible and the decays simply remove angular momentum in intraband cascades. Calculations ${ }^{13}$, based on Monte Carlo simulations of the statistical process, are able to reproduce all the observables connected with the feeding process, namely the SD-band intensity, intensity as a function of spin, the entry distributions (starting points for $\gamma$ deexcitation after neutron emission) and the spectra of $\gamma$ rays feeding the SD bands.

Fig. $8 \mathrm{a}$ from Ref. ${ }^{13}$ shows the measured entry distribution leading to all
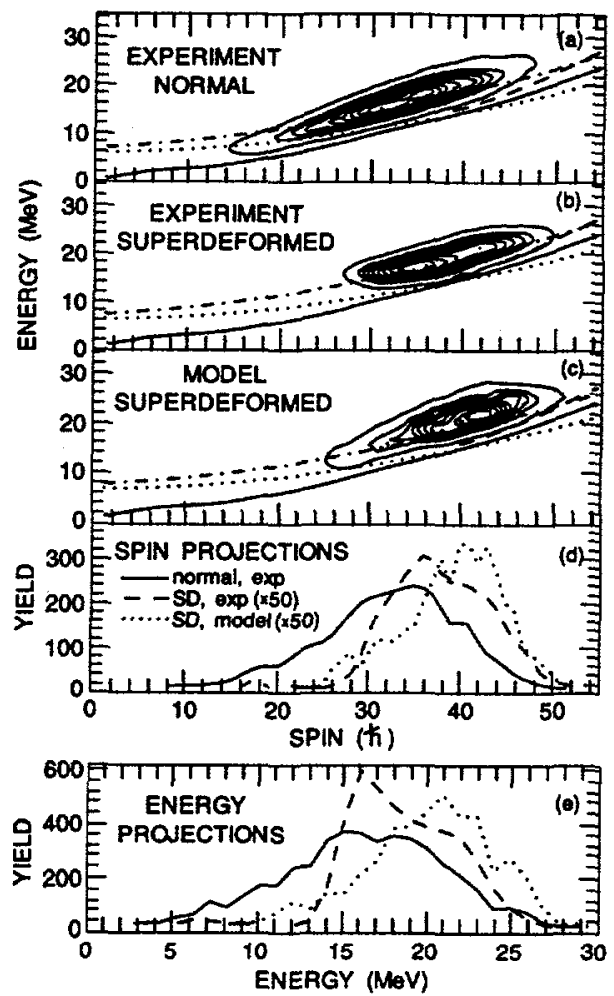

Figure 8: (a-c). Entry distributions in the spin - energy plane leading to the formation of all (mainly normal-deformed) and SD shapes in ${ }^{192} \mathrm{Hg}$. The distributions in $(a, b)$ are measured with the Argonne-Notre Dame BGO array; that in (c) is calculated. (d, e). Projections of the entry distributions in (a-c) on the spin and energy axes. The solid, dotted and dot-dashed lines represent the ND yrast line, SD yrast line and barrier position used in the calculation. From Ref. ${ }^{13}$. 
states of the nucleus ${ }^{192} \mathrm{Hg}$, while Fig. $8 \mathrm{~b}$ shows that the entry distribution for populating a SD band originates from the high-angular momentum portion of the total entry distribution. It is only at large spin that SD states have sufficiently large level density to receive significant population. The entry distribution from SD states is reasonably well reproduced by Monte Carlo simulations (Fig. 8c). The projections of the entry distribution on the spin and energy axes are shown in Fig. $8 \mathrm{~d}$ and $\mathrm{e}$.

A sample of $\gamma$ cascades from events that result in trapping in the SD well is shown in Fig. 9, taken from the results ${ }^{13}$ of the Monte Carlo simulation. Note that successful trapping in the SD well is favored by rapid cooling via

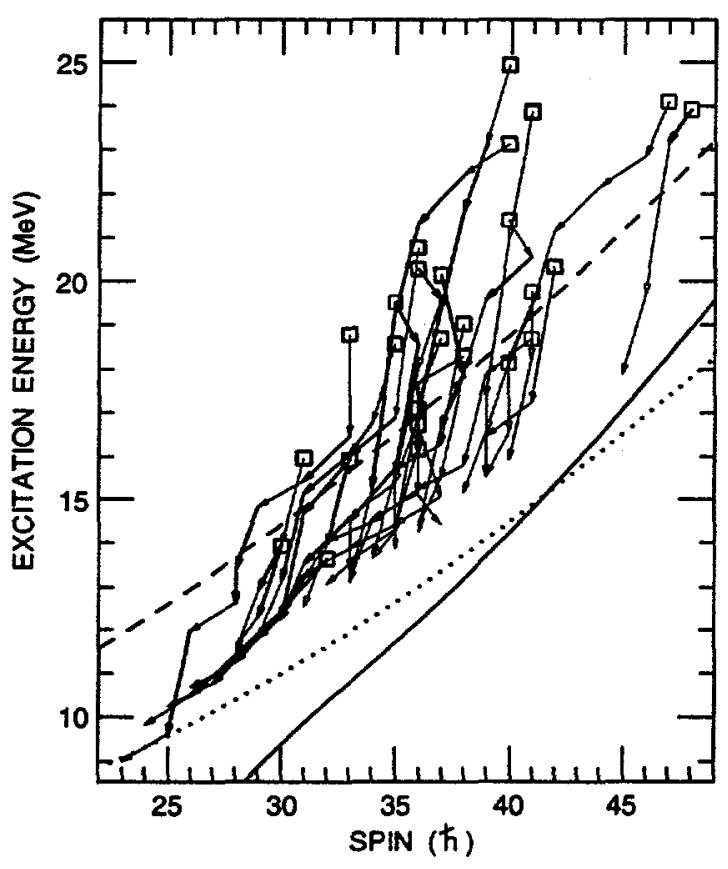

Figure 9: Sample of $\gamma$ cascades that result in trapping within the SD false vacuum, obtained from a Monte Carlo simulation which reproduces all observed feeding properties. From Ref. 13

statistical transitions, which remove energy with little loss in angular momentum. Nuclei that cool rapidly are more likely to survive within the SD well in the competition with tunneling to hot ND states. Collective E2 transitions, which remove 2 units of spin but little internal excitation energy, are emitted in a zone about $1.5 \mathrm{MeV}$ below the barrier and $2 \mathrm{MeV}$ above the SD yrast line. 
In other words, they originate from excited states in the SD false vacuum, thereby providing a probe of the properties and tunneling of these states.

Two of the parameters in the calculations are the well depth W(I) and the excitation energy $E^{*}$ of the SD band above the yrast line. W(I) is assumed to increase linearly from $1 \mathrm{MeV}$ at spin 0 to a value $W(40)$ at spin $I=40$. Values of 3.5-4.5 MeV for $\mathrm{W}(40)$ satisfactorily reproduce the data, suggesting that the well is quite deep at high spin. The SD band intensity is sensitively dependent on the energy $E^{*}$ of the SD states; values of $E^{*}$ between 3.3 and $4.3 \mathrm{MeV}$ at the point of decay satisfactorily reproduced the band intensities. This provided the first indication that the SD bands lie rather high above the ND yrast line in the $\mathrm{Hg}$ region and is consistent with more accurate excitation energies that have been obtained later ${ }^{15}$.

Two important open problems to be investigated are the structure of excited SD bands and the tunneling between hot SD and ND states. Information on these problems comes from the spreading width $\Gamma_{\text {rot }}$ of the rotational strength ${ }^{16}$ of excited SD bands ${ }^{17}$. The spreading width arises from residual nuclear interactions, which couple not only SD states, but also SD and ND states via tunneling. This is an example of tunneling between hot states. Experimental data on the spreading width can be derived from the widths of so-called valleys and ridges in $E_{\gamma}-E_{\gamma}$ correlation plots (see, e.g., Ref. ${ }^{16}$ ). Unfortunately, hitherto there has been little work on excited SD structures or on the tunneling between hot SD and ND states.

\subsection{Cold cascades within SD rotational bands}

In contrast, there is a large body of data on SD bands, since the predominance of effort has been devoted to the relatively more straightforward identification of these bands. When trapping in the SD well occurs, the $\gamma$ cascade cools into or near the bottom of the false vacuum. Isolated by the barrier, the nucleus cools no further and loses angular momentum through cold cascades, with highly-collective E2 transitions ( 2000 Weisskopf Units) connecting members of rotational bands. This phase gives rise to the beautiful spectrum of nearly equal spacings (see Fig. 2), characteristic of collective, ordered rotation. The spacings between transitions yield values of the dynamical moment of inertia $\mathcal{J}^{(2)}$, which have been compared to theoretical values to infer the microscopic structure of the bands. About $175 \mathrm{SD}$ bands have been identified in the mass 150 and 190 regions. However, in all but three cases, the connections of the excited SD bands to the ND yrast states have proven elusive, so that the excitation energies, spins and parities are not known. Lifetime measurements, on the other hand, have been feasible, showing unambiguously that the bands 
have large deformation (see, e.g., Ref. ${ }^{18}$ ). In the mass 190 region, many SD bands have identical quadrupole moments ${ }^{18}$ and most have very close $\mathcal{J}^{(2)}(\omega)$ vs. $\omega$ curves ${ }^{5}$, which slope up with $\omega$. ( $\omega$ is defined as $E_{\gamma} / 2$, where $E_{\gamma}$ is the $\gamma$-ray energy.) The upsloping $\mathcal{J}^{(2)}(\omega)$ curves have been attributed to the alignment of high-j single-particle orbitals and to the presence of pair correlations within the SD well ${ }^{5}$. One surprising discovery has been the phenomenon of identical bands (reviewed in Ref. ${ }^{19}$ ), where many SD bands in neighboring nuclei have either equal transition energies (within $1 \mathrm{keV}$ ) or equal $\mathcal{J}^{(2)}$. Some of the topics mentioned here will be discussed again later.

\section{Decay from SD bands: tunneling out of the false vacuum}

\subsection{Physics from Decay Out of SD Bands}

This section constitutes the main topic of these lecture notes. From investigating the decay out of SD bands we have been able to obtain interesting information on several physics topics. These include: (i) the mechanism for the depopulation of SD bands; (ii) the spin/parity quantum numbers and excitation energies of SD bands; (iii) the microscopic structure of SD bands; (iv) the origin of identical bands; ( $v$ ) the quenching of pairing with increasing temperature; and (vi) the onset of chaos (in ND states) with increasing excitation energy above the yrast line.

\subsection{Decay mechanism}

In this section, we discuss the decay from SD to ND states. Both theoretical and experimental aspects of the decay mechanism are examined.

SD bands exhibit a highly unusual property with decreasing spin: the excitation energy above the ND yrast line increases (Fig. 10), due to their larger moment of inertia. As a consequence, SD states become embedded in a sea of hot ND states with increasing level density. Although SD states are cold and protected from hot ND states by a barrier, the latter squeeze in around the SD state until an inevitable coupling (albeit small) occurs, precipitating a sudden decay.

An important development in understanding the decay mechanism was the work of Vigezzi et al ${ }^{20}$, which laid the theoretical foundation for treating the coupling of isolated SD states with ND states of high level density. The SD state couples, with a spreading width $\Gamma$ to compound ND states, with average level separation $D_{n}$. A SD state can decay via a collective $E 2$ intraband branch (with width $\Gamma_{S D}$ ) to the lower rotational member or, via an admixed compound ND wave function, to lower-lying ND states. A compound state, a complicated 


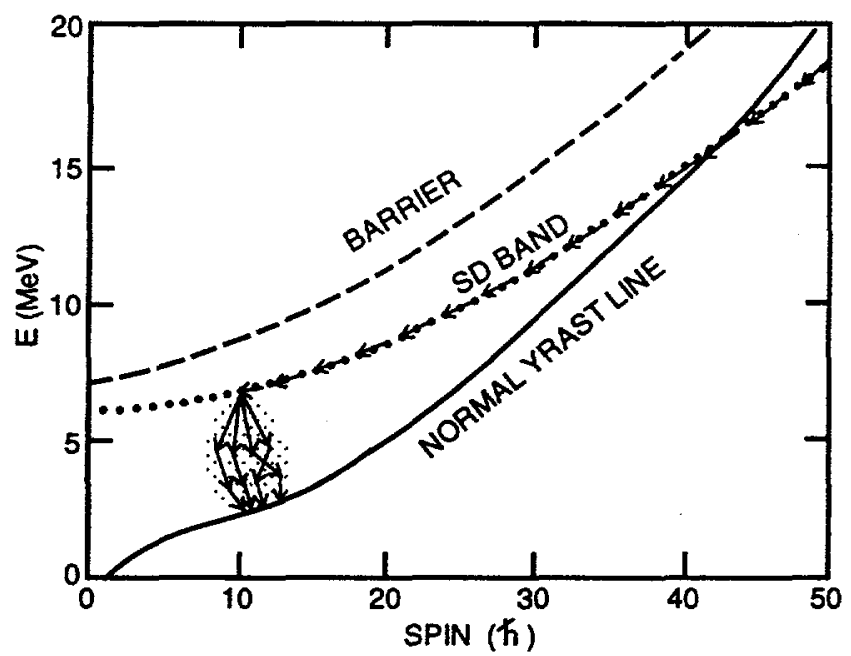

Figure 10: As a SD band loses angular momentum, its excitation energy above the ND yrast line rises. The $\gamma$ cascade is confined in a single path along the SD band, but is very fragmented in the sudden decay from SD to ND states. The approximate barrier separating SD and ND states is shown, which illustrates that the barrier height (separation between barrier and SD band) for tunneling decreases with lower spin.

level with very many components in its wave function, can decay to all states by a statistical process. Its decay rate $\Gamma_{C N}$ can be computed using a $\gamma$ strength function and level densities.

In the Vigezzi model, ${ }^{20}$, the decay rate out of the SD band is governed by one parameter, namely the ratio $\Gamma / D_{n}$. We have applied this model to the decay from $\mathrm{SD}$ bands in the $\mathrm{Hg}$ region, and are able to reproduce the intensities of SD transitions at the point of decay. A value of $\Gamma / D_{n} \sim 0.03$ reproduces the spin at which the decay occurs (Fig. 4). Hence, the spreading width is very small and the SD state couples with the nearest one or two excited ND states. At an excitation energy above yrast of $\sim 4.3 \mathrm{MeV}, D_{n} \sim 0.4 \mathrm{keV}$ giving $\Gamma \sim 13 \mathrm{eV}$, a very small value indeed. A typical nuclear matrix element is $\sim 100 \mathrm{keV}$, four orders of magnitude larger. The SD eigenstate is given as

$$
|S D>+\alpha| N D^{*}>
$$

with $\alpha \sim 0.08$ - a very small value.

The decay out of a SD band occurs through the hot ND component, $\left|N D^{*}\right\rangle$. Although the coupling between SD and ND states is very weak, the decay occurs because $\Gamma_{C N} / \Gamma_{S D} \sim 200$ is large. After a long series of up 
to 20 transitions, this tiny coupling precipitates the rapid decay out of the SD band, a feature which is nearly universal for SD bands. Four factors contribute to the sudden decay. As the spin becomes smaller, $\Gamma_{S D}$ decreases quickly because of its $E_{\gamma}^{5}$ dependence. At the same time, the excitation energy above the ND yrast line grows. This leads to an increase in $\Gamma_{C N}$, as well as a decrease in $D_{n}$, so that the average separation from a ND state becomes smaller. A fourth, and essential, ingredient is an exponential increase of $\Gamma$ with decreasing spin ${ }^{20}$. If $\Gamma$ were to remain constant, only a gradual decrease in SD intensity would be obtained (dashed line in Fig. 4).

When viewed as a tunneling process, $\Gamma$ is associated with a tunneling width. Hence, an exponential increase in $\Gamma$ implies a barrier height $W$ that decreases linearly with diminishing spin. (This trend can be understood from the macroscopic liquid drop term.)

In $A \sim 190$ nuclei, the sudden depopulation of SD bands occurs around spin $8-10$, whereas in the $A \sim 150$ region it happens around spin 25 . Within a given mass region, the level densities, SD excitation energies and the well depths $W(I)$ are roughly the same, so that the decay can happen around the same spin. The fluctuations in decay-out spin (expected because of the stochastic nature of the process) are small since the decrease in W(I) triggers the decay around the same spin values in each mass region.

Shimizu et al ${ }^{21}$ have determined $\Gamma$ or, more specifically, the tunneling action $A(I)$ by computing the well depth and inertial mass as a function of spin. The role of pairing, which increases as the spin becomes smaller, was taken into account in the spirit of the Bertsch hopping model ${ }^{22}$, i.e. by counting the number of level crossings. From our application of the Vigezzi model we can also deduce the $\mathrm{W}(\mathrm{I})$ that reproduce the sudden reduction in SD transition intensities ${ }^{23}$. We can deduce ${ }^{23}$ the action $A(I)=\pi W(I) / \hbar \omega_{B}$, using an assumed ${ }^{20}$ barrier frequency of $\hbar \omega_{B}=0.6 \mathrm{MeV}$. The action inferred from experiment is compared to that calculated by Shimizu in Fig. 11, taken from Ref. ${ }^{23}$. The actions at the point of decay are in reasonable agreement, although the high-spin values and $d A(I) / d I$ are not. Hence, it still remains a challenge to theoretically describe decay out of SD bands.

\subsection{Spectra from Decay out SD States}

It is possible to experimentally confirm the postulate of the Vigezzi model, 20 that the decay occurs via a small admixed component of a hot ND state $\left(\mid N D^{*}>\right.$ in Eq. (1)). In this model the decay spectrum should be just that from a hot compound state, which is schematically illustrated in Fig. 12. A hot state can deexcite by primary (i.e.' first-step) transitions to near-yrast 


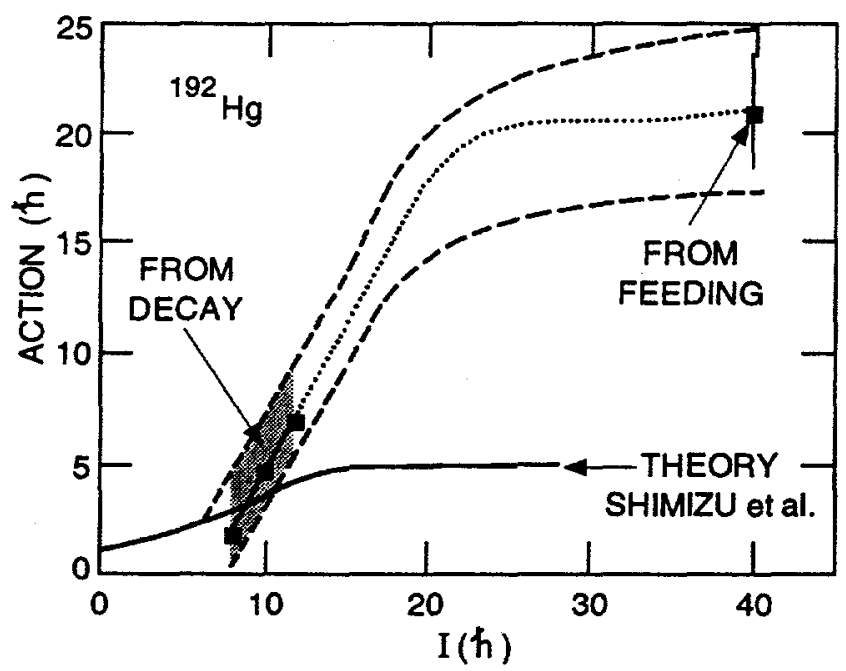

Figure 11: Action $A(I)$ for tunneling between SD and ND states, given as a function of spin. Results from theory ${ }^{21}$ (solid line) and those deduced from experiment (filled squares) are shown. The "experimental" actions around spin 10 are deduced by comparing the SD intensities at the decay point with predictions using the Vigezzi model ${ }^{20}$; that at spin 40 is deduced from the comparison of SD-feeding observables (e.g. that shown in Fig. 7) with model predictions ${ }^{13}$. The deduced actions depend on the assumed level density of ND states; the dashed lines are obtained by multiplying the nominal level density by factors of 10 and 0.1. From Ref. ${ }^{23}$.

states, resulting in sharp $\gamma$ lines. For decay to higher-lying states, with rapidly increasing level density, the lines overlap, form structures, and then smear into a quasicontinuum. Primary transitions to highly excited states of high density, together with the subsequent secondary transitions, form an unresolved background. In addition, transitions between near-yrast states will appear as sharp lines at low energy. These are indeed the features observed in the $\gamma$ spectrum following thermal neutron capture (Fig. 13, from Ref. ${ }^{24}$ ). Hence, if the spectrum from SD decay has these features, it would constitute strong evidence of the postulated decay mechanism.

This spectrum was first obtained in Ref. ${ }^{25}$, where the procedures are described. First, an extremely clean spectrum coincident with a SD band is required and is obtained by setting pairwise coincidence gates on SD lines. A correct background subtraction procedure, such as described in Ref. ${ }^{26}$ is essential. Extraneous events from neutron interactions, coincident $\gamma$ summing and Compton scattering are then removed by the procedures described in Refs. 


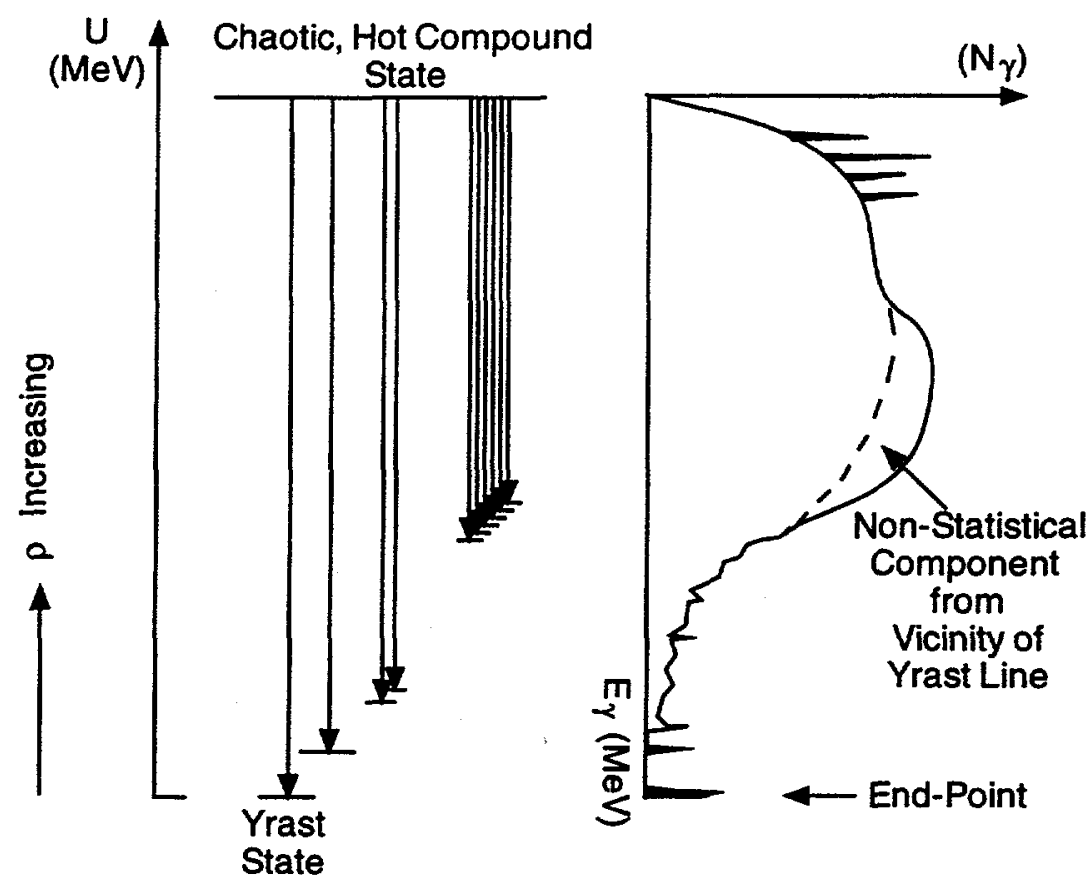

Figure 12: Decay from a highly excited, chaotic, compound state. Some primary transitions to final states at different energies are indicated, together with the characteristic features they yield in the decay spectrum. The sketch of the decay spectrum has a largely statistical form, with some structure coming from secondary transitions occurring near the yrast line. Low-energy peaks are from yrast transitions.

27,28. Corrections for the full-energy peak efficiency and angular distribution effects are then made. Finally, the spectra are put on an "absolute" scale, by normalizing the spectrum so that the ground-state transition (e.g. $2^{+}-$ $0^{+}$transition), has unit area (after correction for conversion electrons). The integral of the spectrum (after correction for coincidence gates) immediately gives the multiplicity, i.e. the average number of steps in the cascade, and the product of multiplicity and average energy gives the total $\gamma$ energy removed.

The spectrum ${ }^{25}$ coincident with the lowest SD band in ${ }^{192} \mathrm{Hg}$ is shown in Fig. 14, together with the spectrum for all states in ${ }^{192} \mathrm{Hg}$, obtained with a coincident gate on the $2^{+}-0^{+}$transition. Differences between the two spectra are immediately apparent, suggesting where the decay-out strength lies in the spectrum. The smooth spectrum in Fig. 14 is a calculation of the feeding statistical spectrum, obtained from a model ${ }^{13}$ that reproduces all observables 


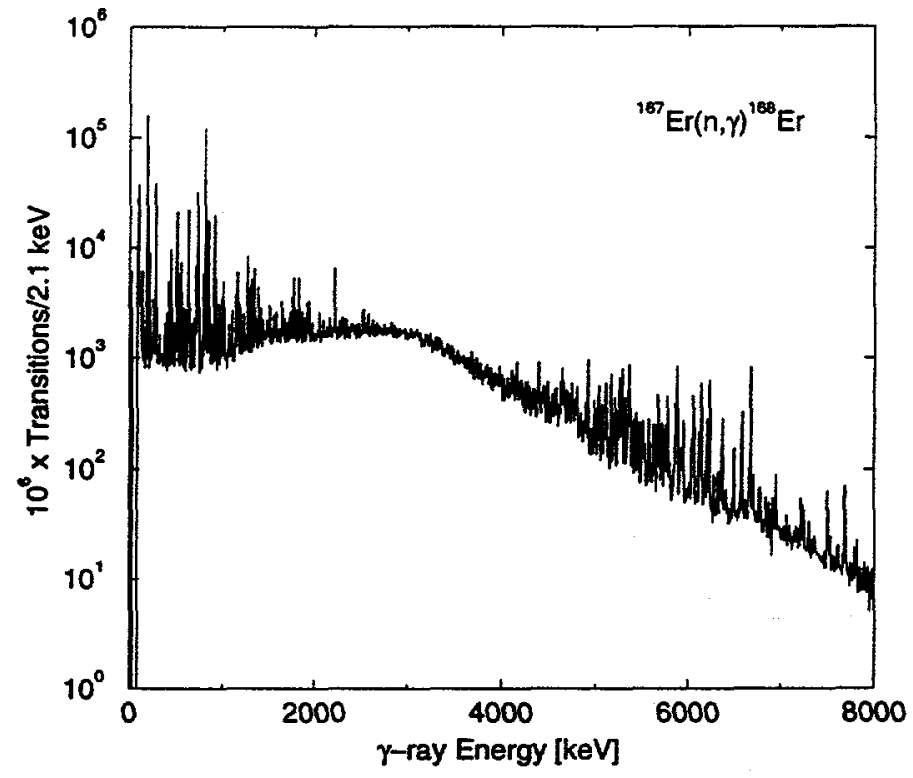

Figure 13: Gamma spectrum following thermal-neutron capture in ${ }^{167} \mathrm{Er}$; from ArgonneBrookhaven-Manchester- collaboration 24 .

in the feeding of SD bands. Subtraction of this statistical spectrum and of the discrete lines yields the spectrum ${ }^{25}$ in Fig. 15. Two feeding components at low energy remain: a large E2 peak (A), arising from excited SD bands, and a M1/E2 peak (B), from transitions immediately preceding feeding of the SD bands. The remaining broad, smooth component represents the $\gamma$ rays connecting SD and ND states; its portion below $\sim 800 \mathrm{keV}$ cannot be unambiguously extracted. The decay spectrum indeed has the features of the statistical spectrum sketched in Fig. 12. The statistical nature of the decay is further demonstrated by its fragmentation over about 1000 primary pathways. 29

A similar decay spectrum ${ }^{30}$, this time from SD band 1 in ${ }^{194} \mathrm{Hg}$, is superimposed on a spectrum ${ }^{24}$ obtained from thermal neutron capture in ${ }^{167} \mathrm{Er}$ in Fig. 16. The end- points in the two spectra are 4.3 and $7.5 \mathrm{MeV}$, respectively, but the dispersions of the spectra are adjusted so that the end-points occur at the same channel in Fig. 16. There is a remarkable similarity in shape and 


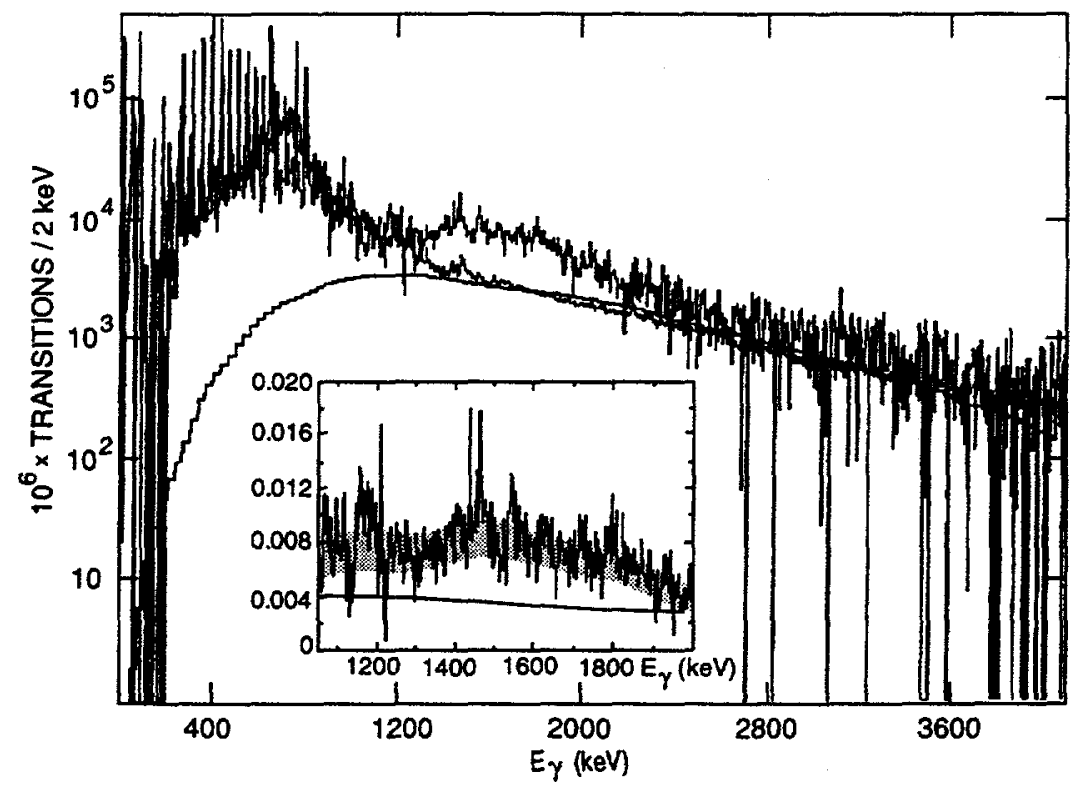

Figure 14: Spectra ${ }^{25}$ obtained from pairwise coincidence gates on SD lines (dark histogram) and from a gate on the $2+-0+$ transition in ${ }^{192} \mathrm{Hg}$ (light histogram). The excess counts around $1.6 \mathrm{MeV}$ suggest significant strength in this region from the decay between SD and ND states. The smooth curve is a calculation of the statistical spectrum feeding the SD band, and is very close to the statistical spectrum (light histogram) feeding ND states. The inset shows a portion of the decay spectrum, revealing that it is composed of lines, overlapping structures and an unresolved component. The integral of each spectrum is its multiplicity.

magnitude between the spectra. (The integral of each spectrum represents the multiplicity.) This remarkable similarity provides the best evidence that decay from SD states is mediated by an admixed component of a compound ND state, and confirms the mechanism suggested by Vigezzi et al. ${ }^{20}$

Sharp peaks at high energy from direct one-step transitions to the yrast line are also expected -see Fig. 12 and 13. Indeed, discrete lines are observed at very high energy $(\sim 4 \mathrm{MeV})$ in the decay spectrum from ${ }^{194} \mathrm{Hg}$ SD band 1 Fig. 17a. These lines, finally observed ${ }^{15}$ after a decade-long search, complete the parallel between spectra from SD and neutron-capture states.

The decay mechanism is now understood as the coupling of a cold SD state to a hot compound ND state. However, the understanding is not complete since quantitatively correct results for the tunneling process still remain a challenge for theory. 


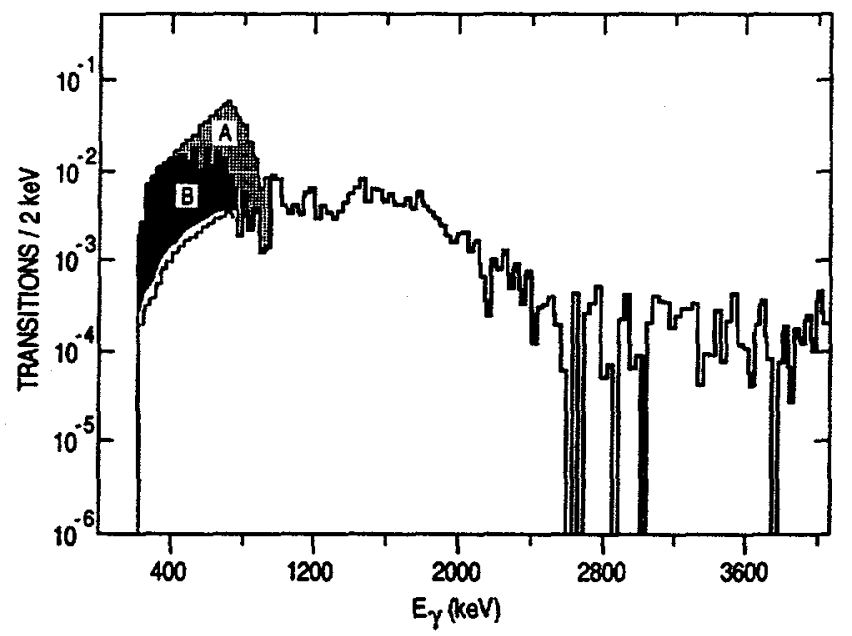

Figure 15: Spectrum from Fig. 14, after subtraction of discrete lines and the calculated statistical spectrum shown there. Components $A$ and $B$ at low energy are from $\gamma$ rays that feed the SD band. The remaining broad component, which has a statistical-like form, represents the decay spectrum connecting SD and ND states. From Ref. ${ }^{25}$.

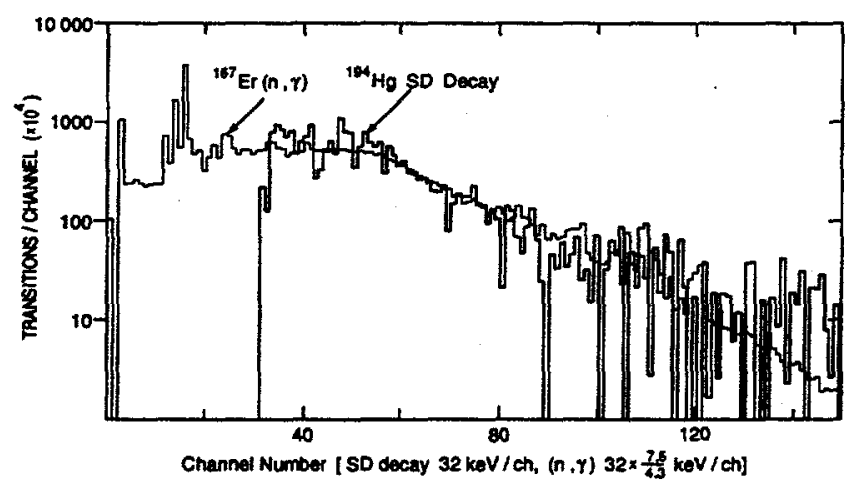

Figure 16: Overlay of spectra from the decay out the ${ }^{194} \mathrm{Hg}$ SD band 1 (thick line) and from thermal-neutron capture (thin line). The spectra have their gains adjusted so that the end-points at 4.3 and $7.5 \mathrm{MeV}$, occur at the same channel. In each case, the integral of the spectrum gives the multiplicity. Note the remarkable similarity in shape and magnitude of the two spectra, showing that the SD decay occurs via a chaotic process, identical to that from thermal-neutron capture. 


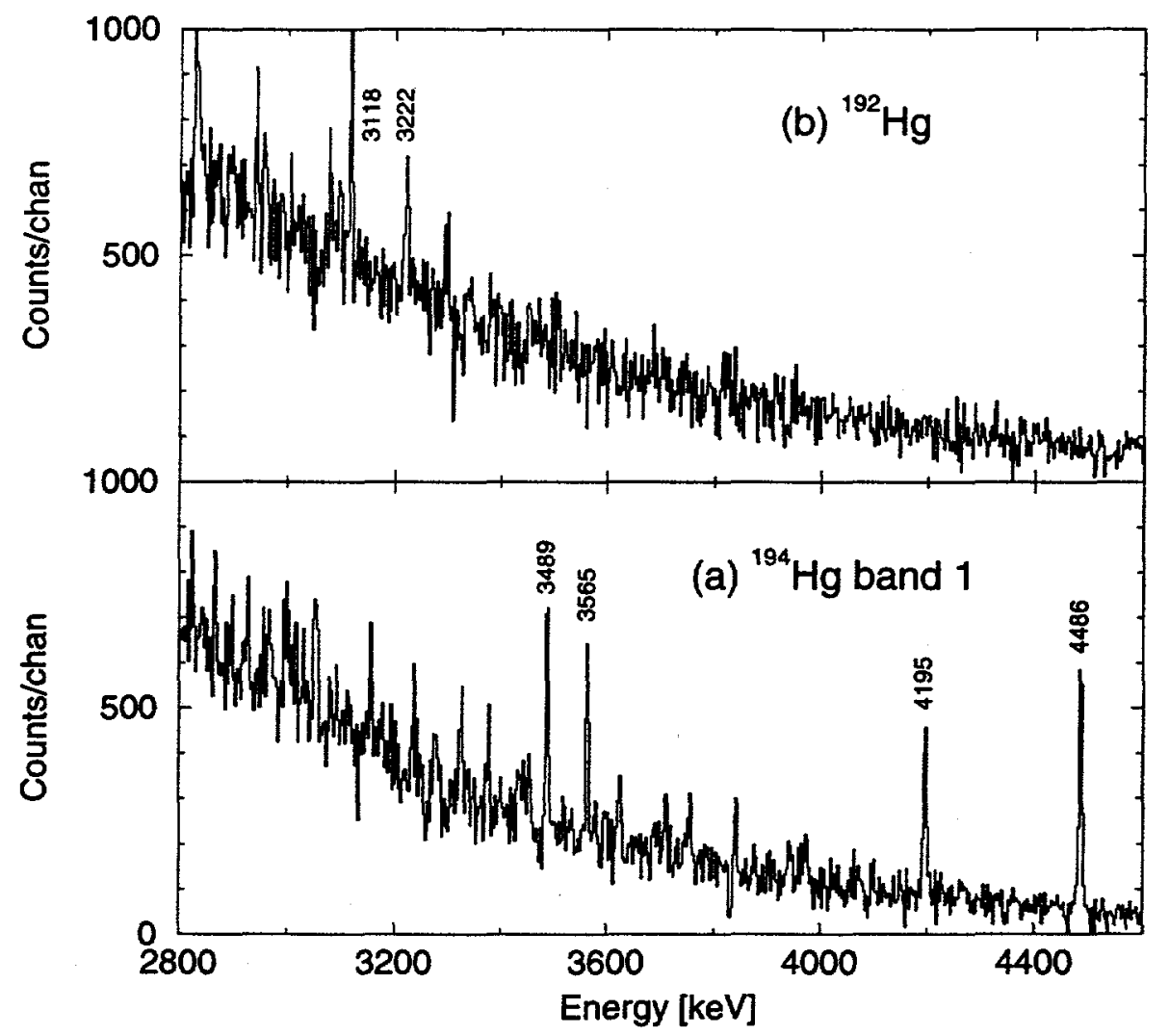

Figure 17: High-energy portions of spectra from (a) ${ }^{194} \mathrm{Hg} \mathrm{SD}$ band 1 and (b) ${ }^{192} \mathrm{Hg} \mathrm{SD}$ band. One-step transitions directly connecting SD and ND states are prominent in (a), but are not seen in (b). The primary lines in (b) represent the first steps of multi-step decays.

\subsection{Spin/parity Quantum Numbers, Energies and Decay Schemes of SD Bands}

After a decade of research in superdeformation and the detection of more than 175 SD bands, the essential quantum numbers and excitation energies are still largely unknown. Some early attempts at finding the decay schemes of SD bands were based on trying to establish coincidences among the highly fragmented decay lines. However, with the realization of the analogy between decays from SD and neutron-capture states, it became clear that the simplest method was to identify the primary lines, which directly connect, in one step, SD and ND yrast states. This led to the discovery ${ }^{15}$ of high-energy, one-step 
transitions in ${ }^{194} \mathrm{Hg}$ - see Fig. 17a. It was a thrilling culmination of a long search!

In addition, pairwise coincidence gates set on a one-step decay line and a SD line made it possible to determine ${ }^{15}$ the exact connections between the lowest SD states (band 1) in ${ }^{194} \mathrm{Hg}$ and the ND yrast states. One-step transitions from an excited SD band in ${ }^{194} \mathrm{Hg}$ have even been found ${ }^{31}$ - see Fig. 18. This figure also displays coincidence spectra that allow definite assignments

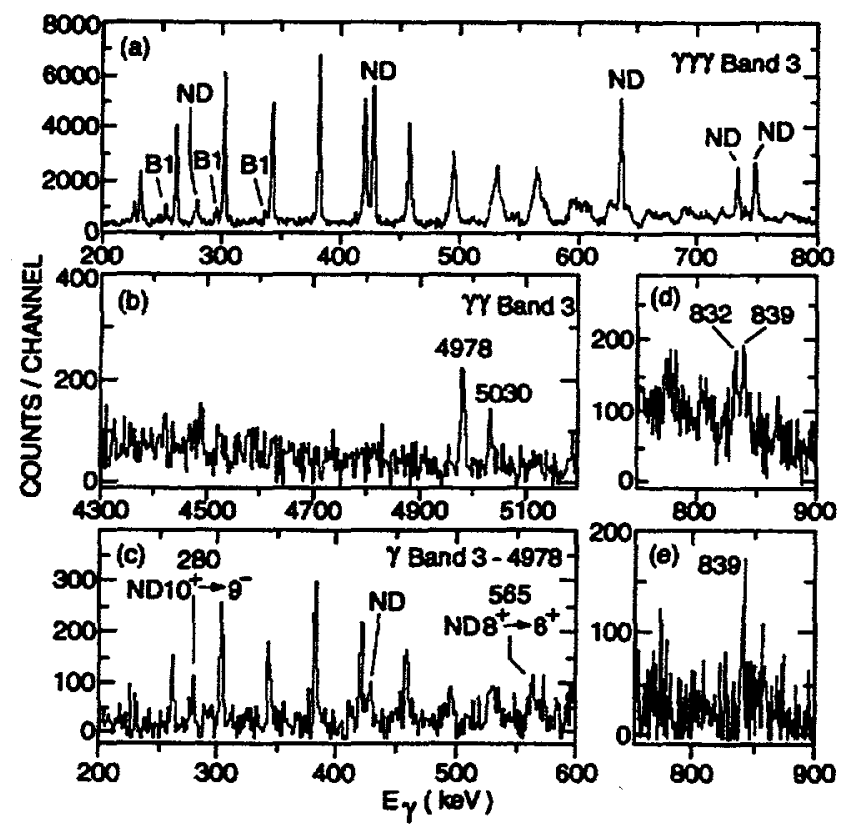

Figure 18: a) Low- and (b) high-energy portions of the spectra coincident with (a) triplets or (b) pairs of transitions from ${ }^{194} \mathrm{Hg}$ SD band 3. (c) Pairwise coincidence spectrum obtained with one gate on the 4978-keV line and another on band-3 transitions, showing transitions - from the $10^{+}$and $8^{+}$ND levels. (d) and (e) Portions of triple- coincidence spectra, showing the 832 and $839-\mathrm{keV}$ interband lines; (d) from a gate on the 343-keV band-3 line and double gates on higher- lying band-3 transitions; and (e) from a gate on the 296-keV band-1 line and double gates on band- 3 transitions.

in a decay scheme of the $\sim 5-\mathrm{MeV}$ one-step lines. A level scheme (Fig. 19) ${ }^{15,31}$ giving the energies, spins and likely parity of two SD bands in ${ }^{194} \mathrm{Hg}$ has been constructed, defining for the first time these quantities for SD bands in the mass 150 or 190 regions. At the point of decay, the excitation energies above the ND yrast states are $\sim 4.3$ and $5.0 \mathrm{MeV}$ for the two bands in ${ }^{194} \mathrm{Hg}$. The 
BAND 1

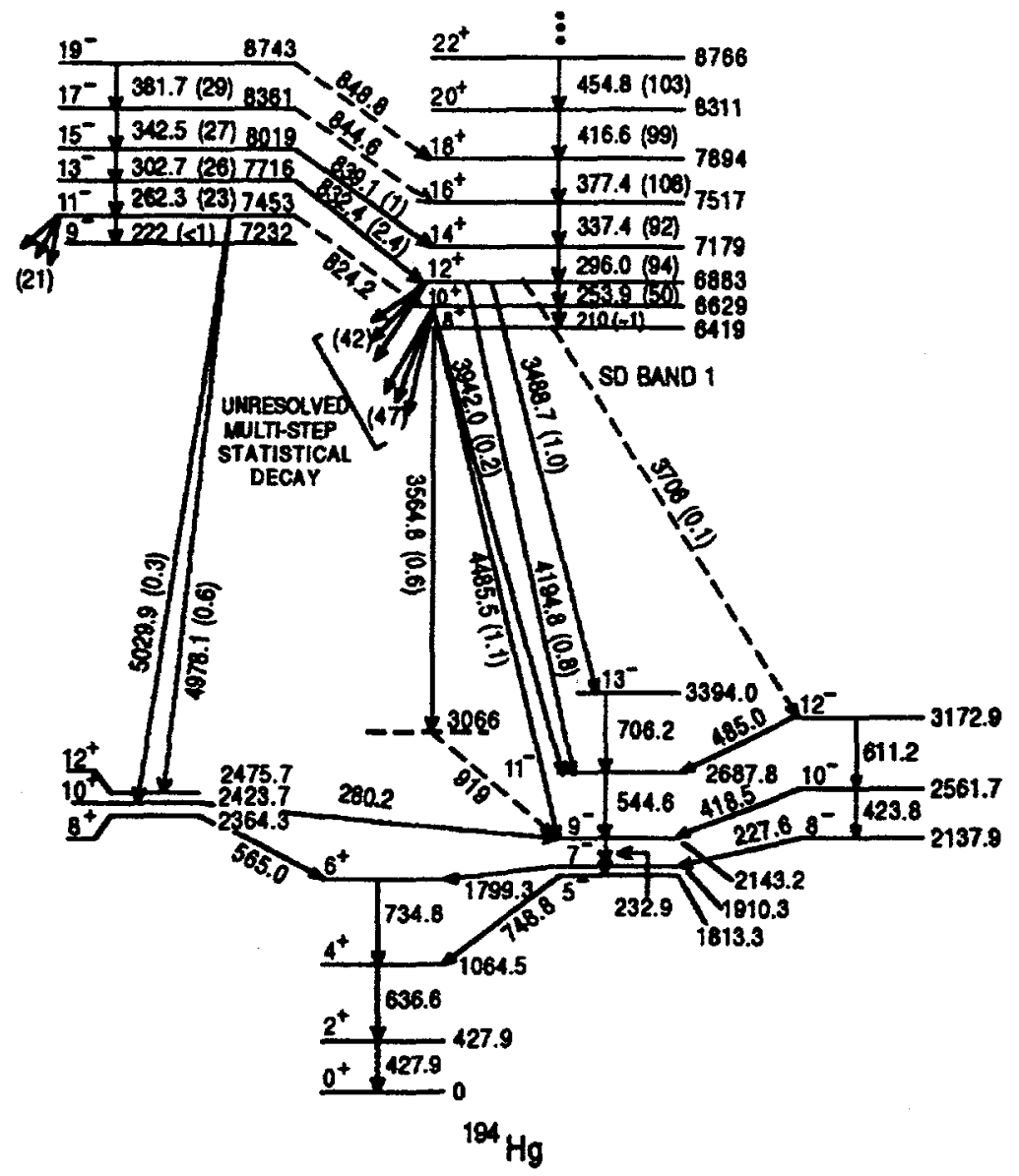

Figure 19: Partial level schemes for the decay of SD bands 1 and 3 in ${ }^{194} \mathrm{Hg}$. Note the 1-step decays from band 3 to both the SD and ND yrast lines. Dashed lines indicate tentative assignments. The transition intensities (in brackets) are normalized to 100 and 30 for the full- strength transitions in bands 1 and 3 , respectively.

SD band in ${ }^{194} \mathrm{~Pb}$ represents the only other case where these quantities are known ${ }^{32,33}$. In this case the excitation energy at the point of decay is lower, $\sim 2.7 \mathrm{MeV}$. 


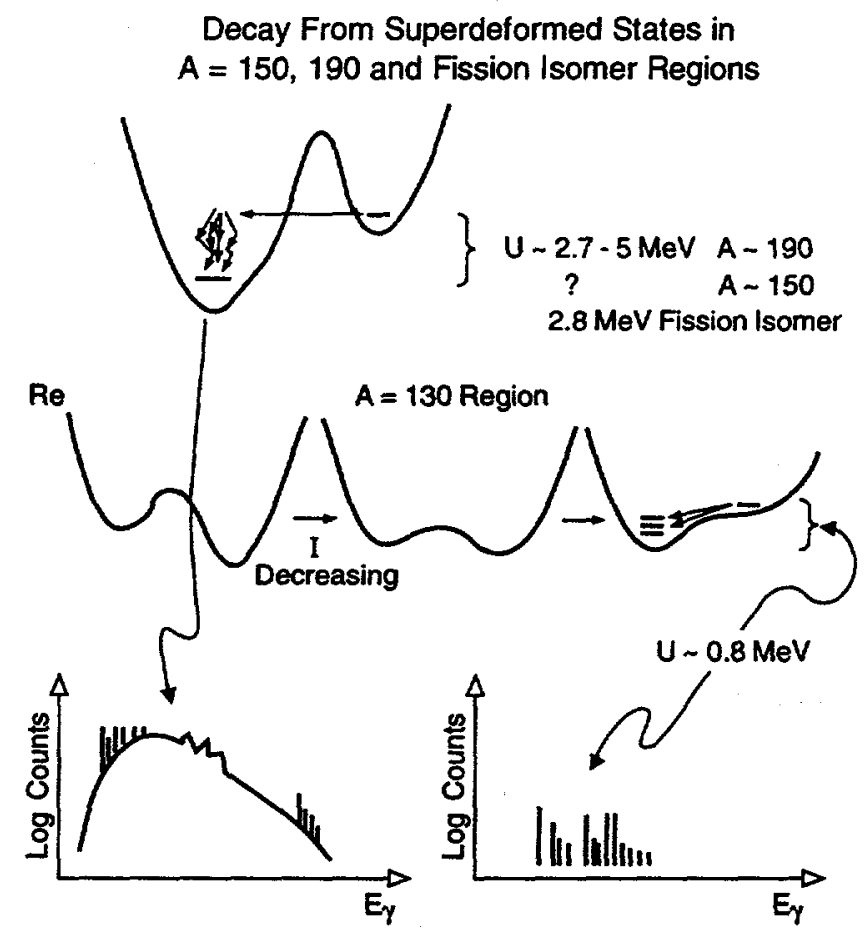

Figure 20: Comparison of decays from SD bands in regions with $A=130,150,190$ and 240, with sketches of the potential energy surfaces, energies of SD states (above ND yrast line) at the points of decay, and cartoons of decay spectra.

We shall now compare the properties of SD states in various mass regions see Fig. 20. In ${ }^{194} \mathrm{Hg}$, which has an excitation energy above yrast of $\sim 4.3 \mathrm{MeV}$, about $10 \%$ of the decay strength is found in discrete primary lines; the rest of the decay proceeds through unresolved $\gamma$ rays which form a quasicontinuum spectrum. In ${ }^{194} \mathrm{~Pb}$, where the excitation energy $(\sim 2.7 \mathrm{MeV})$ is lower, more of the decay is expected as primary lines and, indeed, $\sim 21 \%$ is observed ${ }^{33}$. For the zero-spin fission isomers, which lie around $2.8 \mathrm{MeV}$, the bulk of the decay has been observed as discrete lines in ${ }^{236} \mathrm{U}^{34}$, probably due to the lower level density for low-spin states. The highly-deformed bands that have been located (in spin and energy) ${ }^{35}$ in the mass 130 region lie about $0.8 \mathrm{MeV}$ above the less-deformed states. A substantial fraction ( $50 \%)$ of the decay is in the form of discrete lines in these cases. Here, unlike the other cases discussed so far, the potential energy pocket is much shallower and there is probably 
no barrier separating the states of different deformation at the point of decay. (This may not be the case for bands from which no discrete-line decays have been observed.) In the mass 150 region there is still no SD band whose energy and quantum numbers are definitely known, although the first SD band was observed in ${ }^{152} \mathrm{Dy}$.

The detection and placement of one-step transitions from SD bands has proven to be quite difficult, despite the power of the modern detector arrays. To understand this, one notes that that the decay is a statistical process governed by level densities and a $\gamma$ strength function. Although the one-step lines benefit from their larger energies, they are disfavored by a very small weight in level density. Hence, the bulk of the decay occurs via unresolved $\gamma$ rays from decay to excited states, with high level density, followed by secondary transitions, which together form a quasicontinuum. Therefore, the one-step lines can be favored when the branching ratio for the unresolved component is reduced. This can be achieved by making the phase space for the quasicontinuum component smaller, e.g. by choosing a case with low SD excitation energy (such as ${ }^{194} \mathrm{~Pb}$ ) or a nucleus with a pair gap. The latter, which represents a region above the yrast line with a paucity of levels, is more pronounced in even- even nuclei and when the spin is low. (A further discussion of the role of the pair gap is given later.) These considerations explain the decay properties of SD state in the various regions discussed above. In particular, they account for the increase in the branching ratio for sharp primary lines with smaller SD excitation energy in ${ }^{194} \mathrm{~Pb}$. They also account for the absence of one-step decay lines in the mass 150 region, where the decay occurs around spin 25, where a pair gap is not expected. The statistical nature of the decay has one further ramification: the intensities of the primary lines are subject to fluctuations ${ }^{36}$. With luck, largerthan-average intensities can be obtained; that probably happens in ${ }^{194} \mathrm{Hg}$ and ${ }^{194} \mathrm{~Pb}$. Without luck, the one-step strengths may drop below the detection limit. For example, the high-energy spectrum (Fig. 17b) from the ${ }^{192} \mathrm{Hg}$ SD band, which has similar statistics as that from ${ }^{194} \mathrm{Hg}$ band 1 , does not reveal one-step lines, although other primary transitions, representing the first of several decay steps to the ND yrast line, are visible.

As discussed above, of the $\sim 175$ SD bands which have been found in the mass 150 and 190 regions, there are only three which have finally been precisely located in spin and energy. Figure 18 shows the decay schemes for two SD bands in ${ }^{194} \mathrm{Hg}$. Bands 1 and 3 are the two lowest bands in the SD false vacuum. Band 1, the yrast SD band, has even spin and parity. No signature partner, with odd spin, and equal population intensity has been detected. Hence, the yrast band is just like a $K^{\pi}=0^{+}$"ground" band, but in a false vacuum. The same applies for the yrast SD band ${ }^{32,33}$ in ${ }^{184} \mathrm{~Pb}$. Hence, 


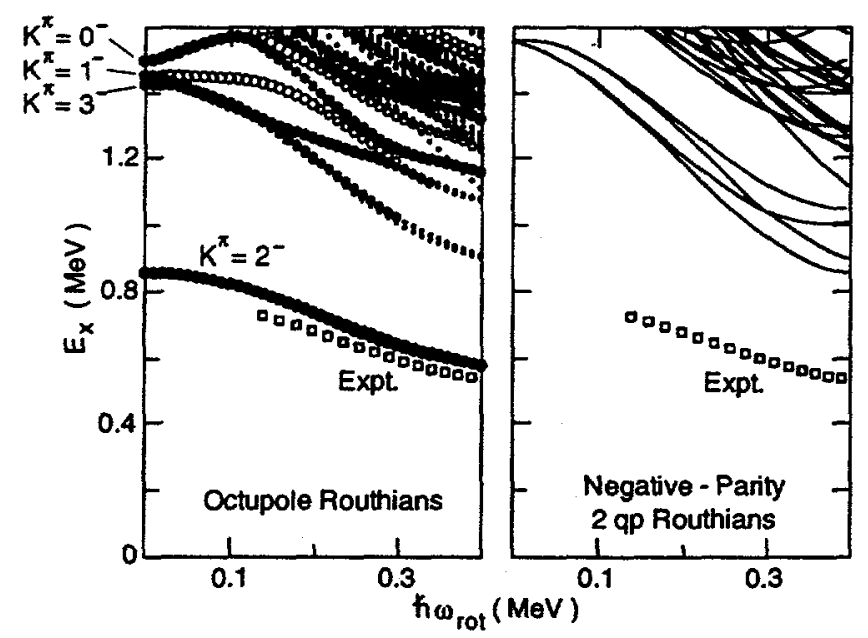

Figure 21: Routhians (orbital energies in intrinsic frame) for theoretical octupole vibrations (open and filled circles) and negative-parity 2-quasiparticle states (lines), compared with experimental data (open squares) for ${ }^{194} \mathrm{Hg}$ SD band 3 .

although the SD states lie at high excitation energy, they are characterized by good quantum numbers $K$ and signature $\sigma . K$, the projection of spin on the symmetry axis, is the quantum number characterizing rotational invariance about this axis. Good signature is a consequence of invariance with respect to $180^{\circ}$ rotation about an axis perpendicular to the symmetry axis. Therefore, the SD states near the bottom of the false vacuum are indeed ordered states, despite their large absolute excitation energies. In contrast, ND states at the same excitation energy probably do not have good $K$ and $\sigma$ (see discussion below).

The excited SD band 3 in ${ }^{194} \mathrm{Hg}$ has odd spin and parity and has been interpreted ${ }^{31}$ as an octupole vibrational band on the basis of its low energy, odd spin and negative parity. Its energy as a function of frequency (Routhian) is very close to that predicted ${ }^{37,31}$ for a $K=2^{-}$octupole vibrational band (see Fig. 21), and is considerably lower than the calculated energies for twoquasiparticle states. Another excited band, band 2 in Ref. ${ }^{6}$, does not have observable one-step decay transitions. However, its transition energies, which are midway between those of band 3 , and its equal population intensity suggest that it is the even spin signature partner of band 3. Low-lying excited SD bands in ${ }^{190} \mathrm{Hg}$ and ${ }^{196} \mathrm{~Pb}$ have also been assigned as octupole vibrational bands. ${ }^{38,39}$ The present body of data supports the predictions of Ref. ${ }^{37}$ that 
octupole vibrations constitute the lowest excited SD bands in even-even mass 190 nuclei. This is a consequence of the intermingling of positive and negative parity levels at large deformation, and implies that the SD shape is reasonably soft with respect to octupole distortion.

${ }^{192} \mathrm{Hg}$ is believed to have closed proton and neutron shells at large deformation. Therefore, the energies of the ND ground and SD states in ${ }^{192,194} \mathrm{Hg}$ and ${ }^{194} \mathrm{~Pb}$ constitute an important triad, which gives the 2-proton and 2-neutron separation energies in both the ND and SD wells. All relevant ND and SD masses are known, with the exception of that for the ${ }^{192} \mathrm{Hg}$ SD band, where there is only a tentative value. The separation energies and SD excitation energies provide an important test of effective nuclear forces, e.g. of different Skyrme interactions for Hartree-Fock-Bogolyubov calculations. Calculations of both separation and excitation energies have been performed ${ }^{40}$ and agree with experimental energies within about $1 \mathrm{MeV}$. It remains a challenge for theory to make more accurate predictions and also for experiment to obtain a definite value for the energy of the SD band in ${ }^{192} \mathrm{Hg}$, for which there is now only a tentative value.

\subsection{Identical Bands}

One of the most exciting discoveries about SD bands is the phenomenon of identical bands, where bands in neighboring nuclei have energies and moments of inertia which are equal for a large span of transition energies. There have been many attempts at explaining the origin of identical SD bands (reviewed in Ref. ${ }^{19}$ ), but the phenomenon is still not understood. There could be a "heroic" explanation, based on the existence of a symmetry (which has yet to be identified). On the other hand, the explanation may be rather "unheroic", based on the accidental cancellations of several effects. Progress in understanding will be made only when there is knowledge of the spins, parities, microscopic structures and quadrupole moments of identical bands. A crucial question to be addressed is whether transitions of equal energies originate from states with identical spins (in even-even nuclei), parities and quadrupole moments.

Band 3 in ${ }^{194} \mathrm{Hg}$ and the vacuum band SD band in ${ }^{192} \mathrm{Hg}$ constitute a pair with transition energies that are equal within $1 \mathrm{keV}$ for a large range of energies ( 382 to $854 \mathrm{keV}$ ). The quadrupole moments of the identical bands in ${ }^{192,194} \mathrm{Hg}$ have been measured to be equal ${ }^{18}$. Unfortunately, the spins and parity of the ${ }^{192} \mathrm{Hg}$ SD band have not been measured. However, it is reasonable to expect that they will both be even, as observed for the vacuum bands in ${ }^{194} \mathrm{Hg}$ and ${ }^{194} \mathrm{~Pb}$. In addition, by using a model-dependent method ${ }^{41,31}$ even spins are also obtained. Hence, there is reasonable confidence that the transitions of equal 
energy are emitted from states with spins differing by $1 \hbar$ and with opposite parity. The difference in the quantum numbers of states emitting identicalenergy transitions may pose a problem for the "heroic" class of explanations. If a symmetry is responsible for identical bands, then the simplest picture would have them share the same quantum numbers.

Insight about the origin of identical bands can be obtained by examining the so-called dynamical and kinetic moments of inertia, $\mathcal{J}^{(1)}$ and $\mathcal{J}^{(2)}$, for the identical bands. $\mathcal{J}^{(1)}=(4 I-2) / E_{\gamma} \hbar^{2}, \mathcal{J}^{(2)}=4 / \Delta E_{\gamma} \hbar^{2}$, where $\Delta E_{\gamma}$ is the energy difference for successive intraband transitions. The $\mathcal{J}^{(1)}$ and $\mathcal{J}^{(2)}$ moments of inertia for ${ }^{194} \mathrm{Hg}$ bands 1,3 and for the ${ }^{192} \mathrm{Hg}$ SD band are shown in Fig. 22 (from Ref. ${ }^{31}$ ). It is significant that $\mathcal{J}^{(1)}$ and $\mathcal{J}^{(2)}$ converge at zero frequency for each of bands 1 and 3 in ${ }^{194} \mathrm{Hg}$. This can be understood from the familiar equations based on the Harris ${ }^{42}$ expansion (with $\hbar=1$ ) of :

$$
\begin{gathered}
\mathcal{J}^{(2)}=d I_{x} / d \omega=\mathcal{J}_{0}+3 \mathcal{J}_{1} \omega^{2} \\
I_{x}=\mathcal{J}_{0} \omega+\mathcal{J}_{1} \omega^{3}+i \\
\mathcal{J}^{(1)}=I_{x} / \omega=\mathcal{J}_{0}+\mathcal{J}_{1} \omega^{2}+i / \omega .
\end{gathered}
$$

The known spins yield alignment $i=0$, so that $\mathcal{J}^{(1)}=\mathcal{J}^{(2)}=\mathcal{J}_{0}$ at $\omega=0$, explaining the convergence of $\mathcal{J}^{(1)}$ and $\mathcal{J}^{(2)}$. Since $\mathcal{J}^{(2)}$ and, hence, $d I_{x} / d \omega$ for ${ }^{194} \mathrm{Hg}$ band 3 is larger than that for ${ }^{192} \mathrm{Hg}$ at low $\omega$ (see Fig. 22a), the difference in $I_{x}(\omega)$ for the two bands grows with $\omega$ at low $\omega$ (Fig. $22 \mathrm{~b}$ ). The larger $\mathcal{J}^{(2)}$ for ${ }^{194} \mathrm{Hg}$ band 3 is largely due to a decrease in pairing $43,44,31$ in the excited SD band, which, as an octupole vibration, is composed of a coherent superposition of 2-quasiparticle excitations. The growth in $\Delta I_{x}$ does not continue, but saturates at 1 because the rate of increase in $\mathcal{J}^{(2)}$ for ${ }^{192} \mathrm{Hg}$ starts to become larger when $\omega>0.15 \mathrm{MeV}$. The latter is due to a larger alignment of particle spin ${ }^{5}$ from high- $\mathrm{N}$ orbitals $(\mathrm{N}=7$ neutrons, $\mathrm{N}=6$ protons) for ${ }^{192} \mathrm{Hg}$ when $\omega>0.15 \mathrm{MeV}$. In other words the unit difference in spin and identical transition energies for the two identical bands result from an accidental cancellation between the effects of pairing and particle alignment. Hence, an unheroic explanation is indicated for this pair of identical bands, for which we have the most complete information. However, a heroic explanation cannot be ruled out since some underlying symmetry may be responsible for the cancellation. However, neither the symmetry nor its cause have been identified, so that the case for a heroic explanation, alas, needs bolstering.

\subsection{Quenching of Pairing with Excitation Energy}

The $\gamma$ decay out of SD bands is a statistical process, which samples and probes all of the lower-lying ND states. Therefore, the decay spectrum is sensitively 


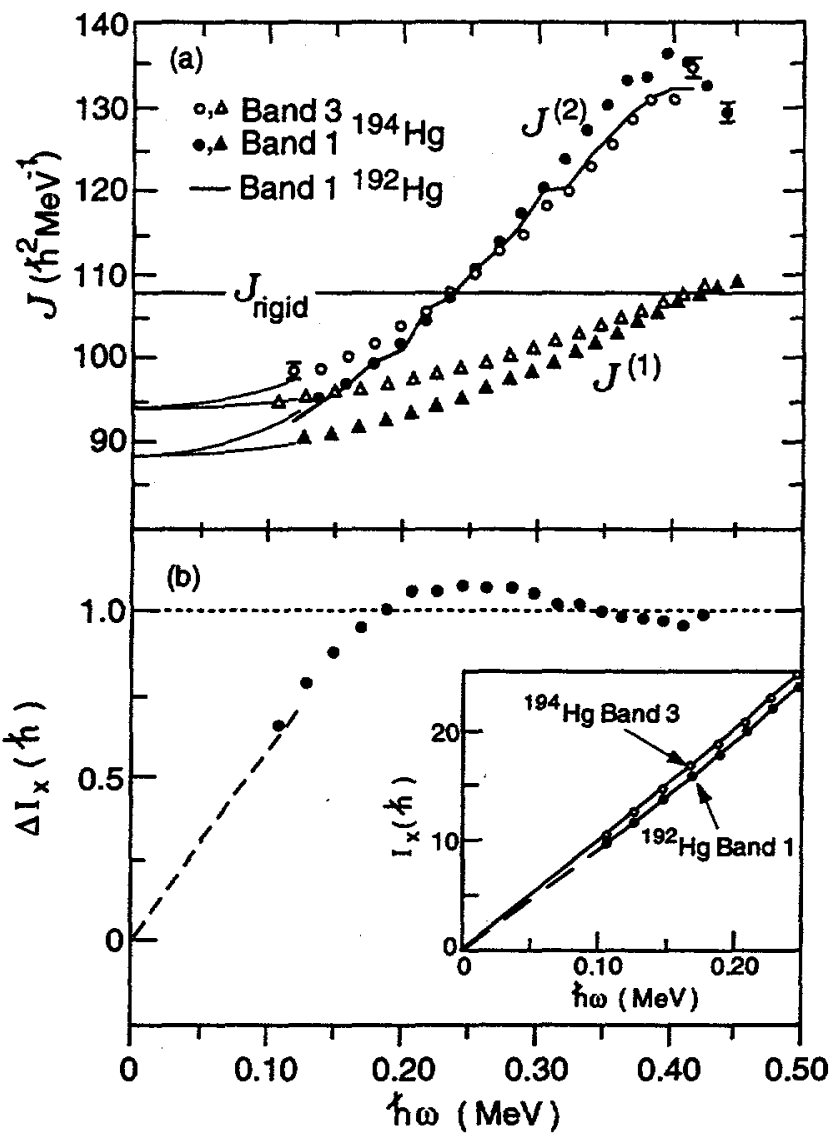

Figure 22: (a) $\mathcal{J}^{(2)}$ (circles) and $\mathcal{J}^{(1)}$ (triangles) moments of inertia for bands 1 (filled symbols) and 3 (open symbols) in ${ }^{194} \mathrm{Hg}$. The extrapolations to zero frequency (thin lines) are from fits to Eq. 2 and Eq. 4. The solid line shows $\mathcal{J}^{(2)}$ for the vacuum SD band in ${ }^{192} \mathrm{Hg}$. (b) $\Delta I_{x}$ vs. w. $\Delta I_{x}=I_{x}\left({ }^{194} \mathrm{Hg}\right.$ SD band 3$)-I_{x}\left({ }^{192} \mathrm{Hg}\right.$ SD band 1$)$ and $I_{x}=\sqrt{\left[I(I+1)-K^{2}\right]}$, where $K=2$ and 0 , respectively, for the two bands, which have identical transition energies for $\omega>0.17 \mathrm{MeV}$. The dashed line shows an extrapolations using Eq. 3. Inset: $I_{x}$ for the two SD bands.

dependent on the density of states. The latter is controlled by pair correlations and by the damping of these correlations with excitation energy. Hence, the decay spectrum provides a probe of pairing ${ }^{45}$. This unanticipated application of the study of the decay process is explained pictorially in Fig. 23. The figure 
also contrasts how the pair gap $\Delta$ quenches with temperature in a macroscopic

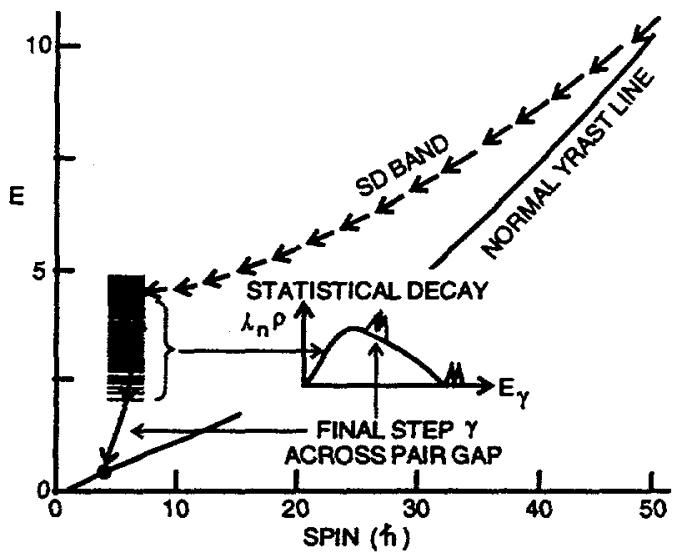

Decay from "single sharp" exclted state a sensitive probe of:

level density $p(U)$

stepwise reduction of pair gap $\Delta$
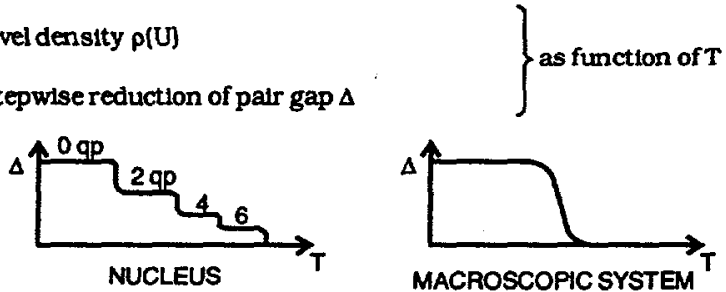

Calculations of - D $\phi s s i n g$ et al

self-consistent $0,2,4,6$... quasiparticle energles, particle no, projectlle

$\rho(U)$

diagonalization

rcascades

spectrum

Figure 23: Illustration of how the decay from an excited state can probe properties of lowerlying states ${ }^{45}$. The decay spectrum is governed by the level densities, which are controlled by pair correlations. The decrease in pair gap $\Delta$ with temperature $T$ in a nucleus and in a macroscopic superfluid are also compared.

or mesoscopic superconductor. In the former, the decrease in $\Delta$ is rather abrupt. However, one would expect the drop to be smeared out in the latter due to pairing fluctuations. The predicted ${ }^{45}$ decrease in $\Delta$ is even more interesting, with stepwise drops corresponding to increasing numbers of quasiparticle pairs.

Døssing et al. ${ }^{45}$ have used a self-consistent BCS treatment of pairing, with particle-number projection and diagonalization, to calculate the energies of quasiparticle levels. Based on these'levels, the $\gamma$ spectrum in statistical 
decay from a sharply defined state was also calculated and compared with the experimental decay spectrum (Fig. 24a). The theoretical spectrum with $\Delta=0.7 \mathrm{MeV}$ reproduces the main features of the experimental spectrum,
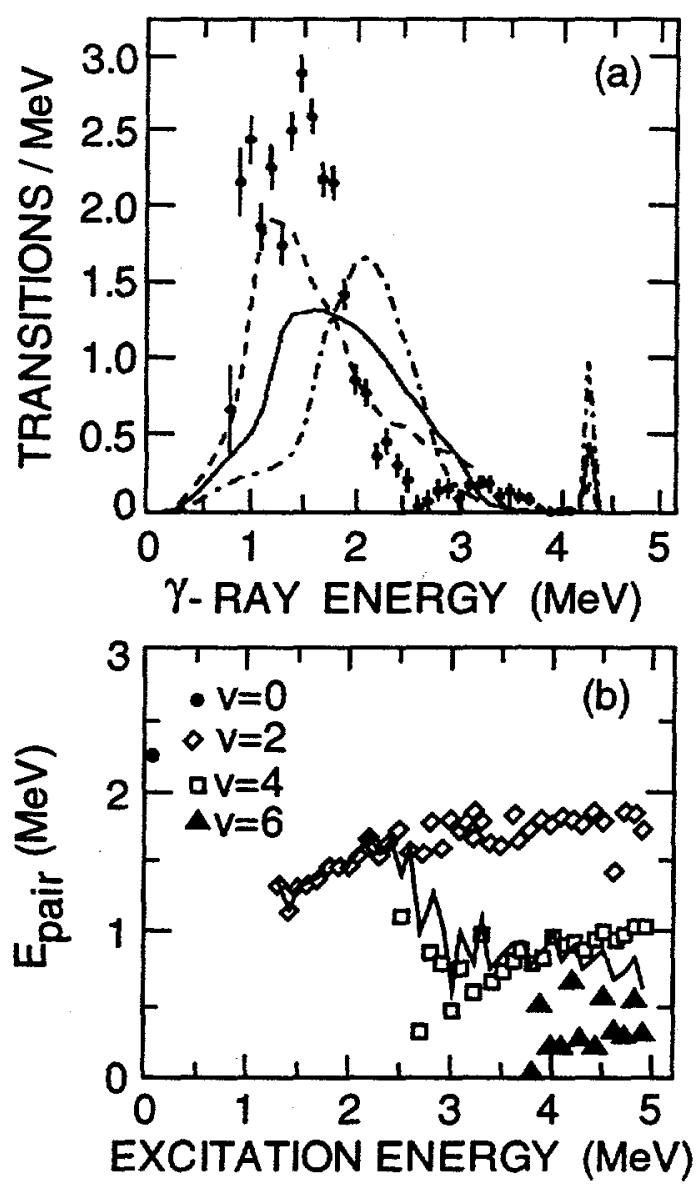

Figure 24: (a) Statistical spectra of an even-even nucleus from an excited state at $U=4.3$. Lines are calculated with levels obtained from BCS theory, with particle-number projection and diagonalization. Dashed, solid and dot-dashed lines correspond to different pairing strengths, parameterized by gaps $\Delta=0.7,0.9$ and $1.1 \mathrm{MeV}$. The spike at $4.3 \mathrm{MeV}$ represents a one-step transition. Data points with errors represent the spectrum from decay out of the ${ }^{192} \mathrm{Hg}$ SD band, which could be extracted only above $0.8 \mathrm{MeV}$. (b) Pair correlation energy for $\Delta=0.9 \mathrm{MeV}$. The points are for $0-, 2-, 4-$ and 6 - quasiparticle states; the line is the average value. From Ref. ${ }^{45}$. 
namely the "squeezing" of the spectrum around $1.6 \mathrm{MeV}$, the depletion in yield at low energy, and the occurrence of one-step high-energy transitions. The pair gap has a significant consequence: the last step in the $\gamma$ cascade has to vault the gap (see Fig. 23), leading to a concentration of strength around 1.6 MeV. Another distinctive feature is also predicted, namely a depletion in yield extending $\sim 1.6 \mathrm{MeV}$ below the one-step lines, from the lack of primary decays into the pair gap. However, this depletion is difficult to observe.

The creditable reproduction of the experimental spectrum (Fig. 23a) lends confidence to the theoretical prediction about the decrease in pairing energy $E_{p a i r}$ as a function of excitation energy. The stepwise decrease in $E_{\text {pair }}$ from the onset of 2- and 4- quasiparticle excitations is evident in Fig. 24b (circle and solid line). At higher energy, remnant pairing energy persists and any further stepwise decrease is washed out. The combination of theory and experiment provides the first plausible look at the quenching of pairing with temperature in a nucleus - many decades after the first recognition of the occurrence of nuclear pairing. This example also illustrates the synergistic collaboration of experiment and theory. An important theoretical step for comprehending the properties of excited nuclei is the calculation of the same quantity as is measured, namely the spectrum.

\subsection{Onset of Chaos with Excitation Energy}

Another unexpected application of the decay of SD states is as a probe of the onset of chaos (in ND states) with excitation energy. Cold states near the yrast line are characterized by order, quantum numbers and selection rules. In contrast, highly-excited states near the neutron separation energy, $\sim 8 \mathrm{MeV}$, are known to be chaotic from the distribution of nearest-neighbor spacings 9 and from the fluctuation properties ${ }^{36}$ of neutron- and $\gamma$ - decay widths. ${ }^{46,47}$ How does the transition from order to chaos occur with increasing energy? Is chaos present at intermediate energies, e.g. between 2.5 and $5 \mathrm{MeV}$ ? In this range, the decaying SD state provides a sharp probe state for determining chaoticity. (It is otherwise very difficult to obtain a highly-excited sharp state.)

One signature of chaos is a breakdown of selection rules in the electromagnetic decay of excited states, leading to a fluctuation and, hence, a distribution in transition strength. A well-known example is the Porter-Thomas distribution, ${ }^{36}$

$$
P_{S}=(2 \pi S \bar{S})^{-1 / 2} \exp (-S / 2 \bar{S}),
$$

which describes the fluctuations in neutron- and $\gamma$ - decay widths $S$ near the neutron threshold ${ }^{46,47}$. (The fluctuations provide a more direct measure of the complexity of the wave function than the nearest neighbor spacings.) The 
contrast between ordered decay, governed by selection rules imposed by quantum numbers, and chaotic decay, governed by level densities and fluctuating strengths, is illustrated for the different decay modes of SD states in Fig. 25.

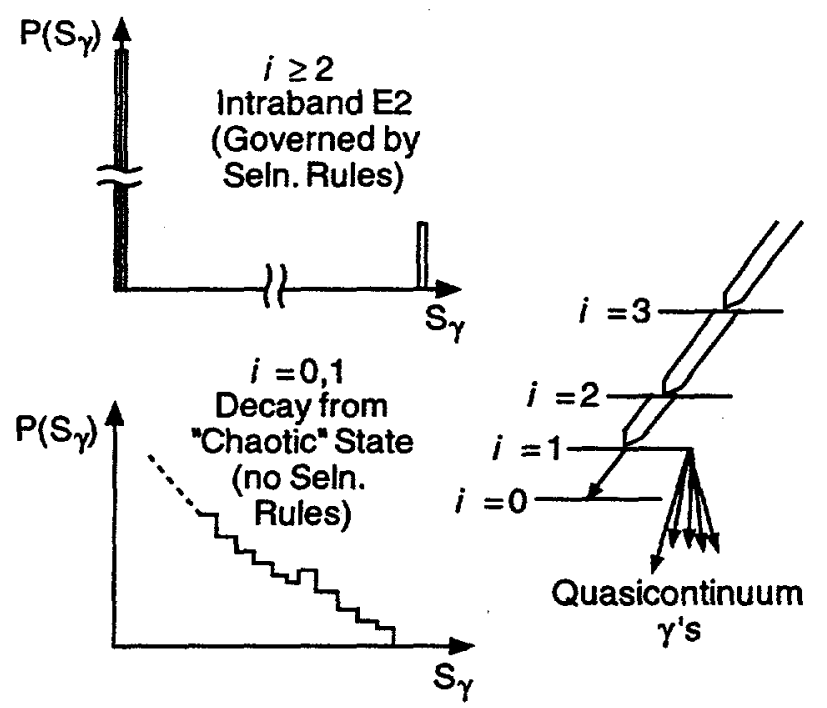

Figure 25: Decay modes of SD levels. Levels with $i<1$ decay to ND states, those with larger $i$ deexcite to band members. The distribution of reduced transition strengths $S_{\gamma}$ for the two cases are illustrated. Decays from states with $i \geq 2$ are governed by selection rules, with population of only one state, with the same $K$ quantum number. Primary-transition strengths for $\mathrm{i}=0,1$ display fluctuations, which has a Porter-Thomas form ${ }^{36}$ in the chaotic limit.

The statistical-like form of the decay spectrum out of SD states suggests that the admixed ND excited state is quite chaotic. Further evidence is provided by the fluctuating strengths of primary transitions from the SD state. $A$ qualitative illustration of fluctuations is the contrast in the intensities of the one-step lines (Fig. 17) in ${ }^{192,194} \mathrm{Hg}$; Fig. 17 shows that these are strong in ${ }^{194} \mathrm{Hg}$, but are undetectably weak in ${ }^{192} \mathrm{Hg}$. Quantitative evidence is provided 48 in Fig. 26, which shows the distribution in the reduced transition strengths of primary transitions from the component $\left|N D^{*}\right\rangle$ in the eigenfunction of the SD state. This distribution is consistent ${ }^{48}$ with the Porter-Thomas form, shown as a dashed line in Fig. 26. In the fully chaotic limit, the fluctuations should indeed have a Porter-Thomas distribution. However, we cannot yet draw a definite conclusion that this limit is attained, since the primary transitions are so weak that we can detect only strong transitions in the tail of the 


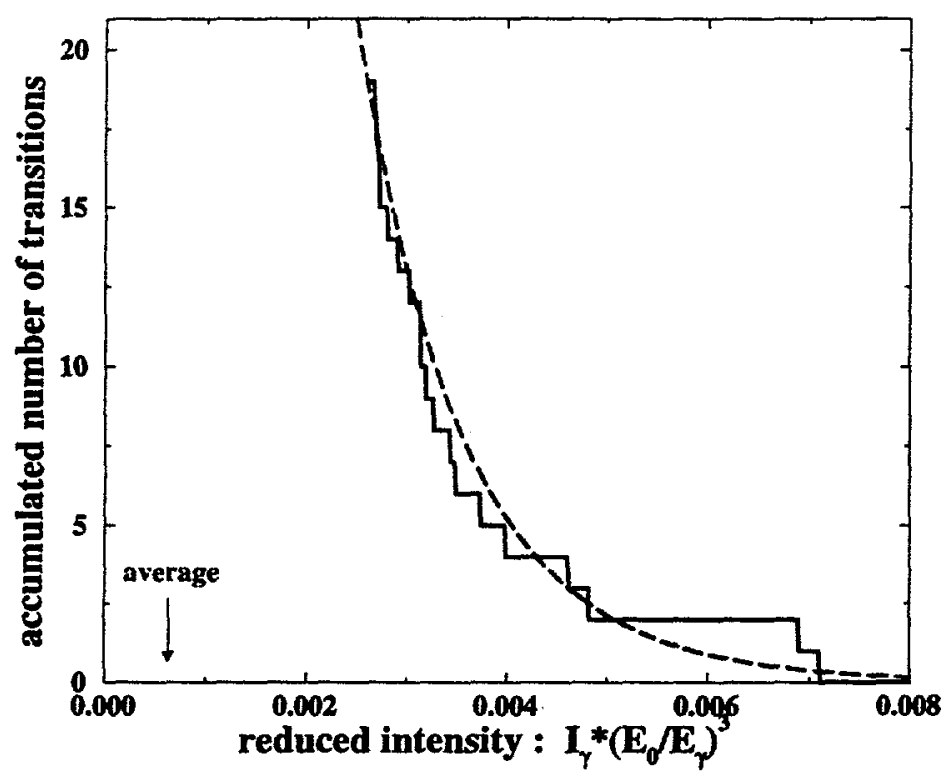

Figure 26: Cumulative number of primary transitions vs. reduced transition strengths, $I_{\gamma}\left(E_{0} / E_{\gamma}\right)^{3}$, from ${ }^{194} \mathrm{Hg}$ SD band 1. (The counting starts from the most intense transition.) The dashed line shows the cumulative distribution expected from a Porter-Thomas distribution that fits the data; its average value is indicated. From Ref. ${ }^{48}$.

distribution. Nevertheless, it is already clear that ND states $\sim 4.3 \mathrm{MeV}$ above the yrast line are quite chaotic - they are certainly not ordered - but we are still in the process of trying to quantify the degree of chaoticity. Furthermore, some open questions remain, e.g. it is not understood why ${ }^{194} \mathrm{Hg}$ is so "cooperative" in revealing the one-step lines from two of its three SD bands.

The long-term goal is to understand how quantum numbers damp out with excitation energy, as reflected in $\Gamma_{Q} / D$, where $\Gamma_{Q}$ is the spreading width of states with a particular quantum number, e.g. $K$, and $D$ is the average level spacing. Another aim is to understand how the good quantum numbers $(K, \sigma)$ of the SD state dissolve upon tunneling from the false into the true vacuum. 


\section{Some Other Examples of Tunneling in Nuclear Physics}

The nucleus offers other opportunities for investigating tunneling. A few examples from recent work will be briefly mentioned here.

\section{$4.1 \Delta I=4$ Bifurcation in $S D$ Bands}

SD intraband transitions have energies that smoothly increase with spin and have almost equal spacings. This high degree of regularity makes it possible to inspect for small excursions from a perfectly smooth behavior. The first example of a small irregularity was found ${ }^{49}$ in the SD band of ${ }^{149} \mathrm{Gd}$. Transitions from states with $\Delta I=4$, i.e. every other transition, were found to be slightly displaced by $\sim 500 \mathrm{eV}$ with respect to the other set with intermediate spins. The small oscillatory behavior ${ }^{49}$ in ${ }^{149} \mathrm{Gd}$ is displayed in Fig. 27 (from Ref. ${ }^{50}$ ). Two other examples have been found in ${ }^{148} \mathrm{Eu}$ (yrast) and ${ }^{148} \mathrm{Gd}$ (excited SD band 6)- see Fig. 27. There are only very few examples of such oscillations which have been confirmed; the vast majority of bands do not exhibit this behavior, as typified by the yrast SD band in ${ }^{148} \mathrm{Gd}$ (yrast). It is interesting and significant that the three bands that show $\Delta I=4$ bifurcation have either identical transition energies or moments of inertia $\mathcal{J}^{(2)}$. Perhaps the Hamiltonians for the three bands all share some common feature (possibly a symmetry).

Several explanations for the $\Delta I=4$ bifurcation have been proposed. An interesting suggestion, in which tunneling is responsible for the observed oscillations, was advanced by Hamamoto and Mottelson. ${ }^{51}$ The rotational Hamiltonian has the general form:

$$
H_{\text {rot }}=A_{0} \bar{I}^{2}+A I_{3}{ }^{2}+B_{1}\left(I_{1}{ }^{2}-I_{2}^{2}\right)+B_{2}\left(I_{1}{ }^{2}+I_{2}{ }^{2}\right)
$$

$\bar{I}$ is the angular momentum vector, $I_{i}$ denote its three components in the body-fixed coordinates of the deformed nucleus, and the $A$ and $B$ terms are parameters. Hamamoto and Mottelson pointed out that under special conditions, namely $A \sim 100 B_{1}, B_{2} \sim 0$, regular oscillations of the type observed in ${ }^{149} \mathrm{Gd}$ can be obtained. These conditions imply a small symmetry breaking of $Y_{44}$ type around the symmetry axis. This gives rise to a four-fold symmetry about this axis, so that $\bar{I}$ is trapped in one of four minima. Tunneling between adjacent-minima splits the degenerate states. If the tunneling matrix element changes sign regularly each time $|\bar{I}|$ increases by 2 , alternate shifts in energy can be obtained. While it has been recognized that one particular form of the Hamiltonian can give rise to the observed oscillations, the conditions for obtaining this form are not understood. In other words, the underlying reason for getting the oscillations in the particular three cases is still not known. 


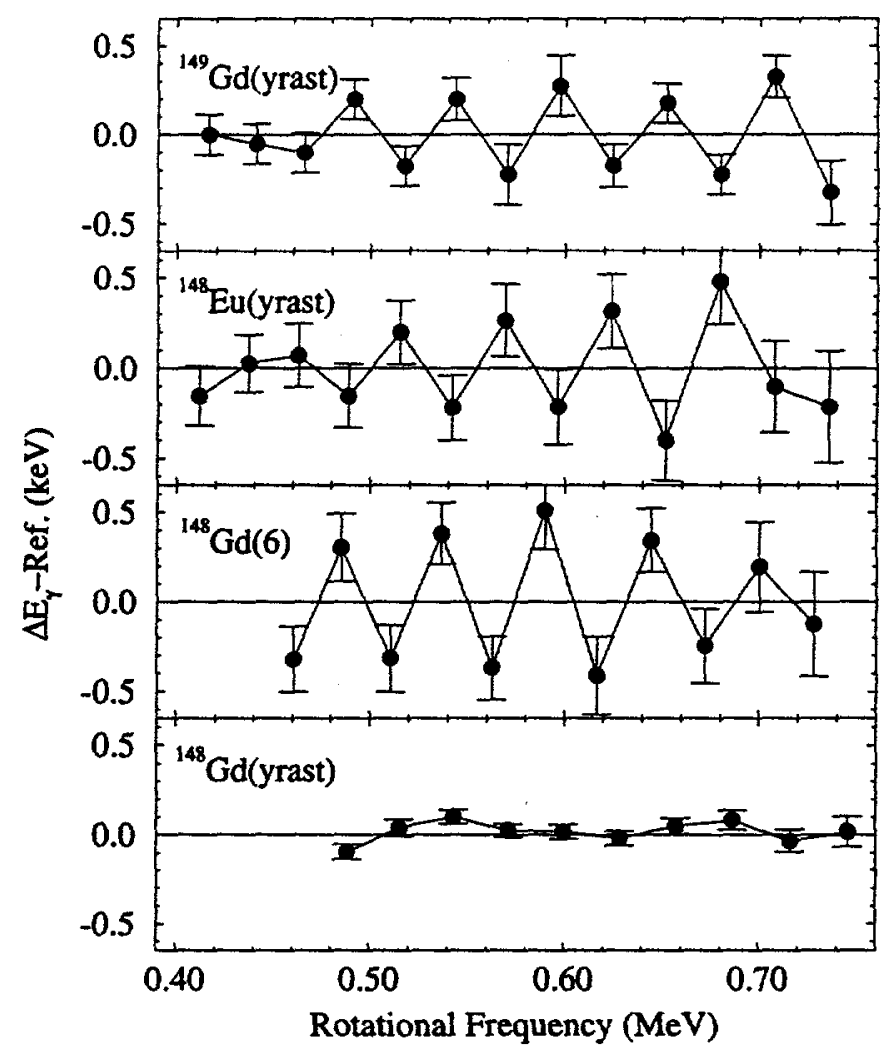

Figure 27: Deviations of transition energies from a smooth "fit" of the measured transition energies in four SD bands. Three bands, which have identical $\mathcal{J}^{(2)}$, exhibit $\Delta I=4$ bifurcation, but most bands are smooth, as exemplified by the spectrum in the bottom panel. From Ref. ${ }^{30}$

\subsection{Sub-barrier Fusion}

It has been known since the 1970's that nuclei can fuse even though there is insufficient energy to surmount the Coulomb barrier. A particularly interesting example is the production ${ }^{52}$ of element 110 with the reaction ${ }^{64} \mathrm{Ni}+{ }^{208} \mathrm{~Pb}$; at the optimal energy, the surfaces of the fusing nuclei are still separated by $\sim 2 \mathrm{fm}$ at the point of closest approach. Nevertheless, in this case, and many others, fusion below the barrier is observed. The fusion is enabled, in one view, by fluctuations of the barrier height brought about by coupled-channel effects from inelastic excitation in the target and/or projectile. An alternative 
view is that surface vibrations, induced by the polarizing effects of the two ions, provides the pathway to fusion. In other words, not only is the radial separation $r$ between the ions important, deformation degrees of freedom $\alpha$ also play a role - giving an example of tunneling in a multidimensional $(r, \alpha)$ space. This subject is discussed in recent reviews of sub-barrier fusion ${ }^{53,54}$.

\subsection{Proton Emitters}

Proton-rich nuclei beyond the line of stability can exhibit proton radioactivity ${ }^{55}$. The ground states of these nuclei are proton unbound, and decay by emission of protons. Recent work at Argonne ${ }^{56}$ has added many new proton emitters - see Fig. 28. Using powerful new experimental techniques in conjunction with a Fragment Mass Analyzer for unambiguously identifying

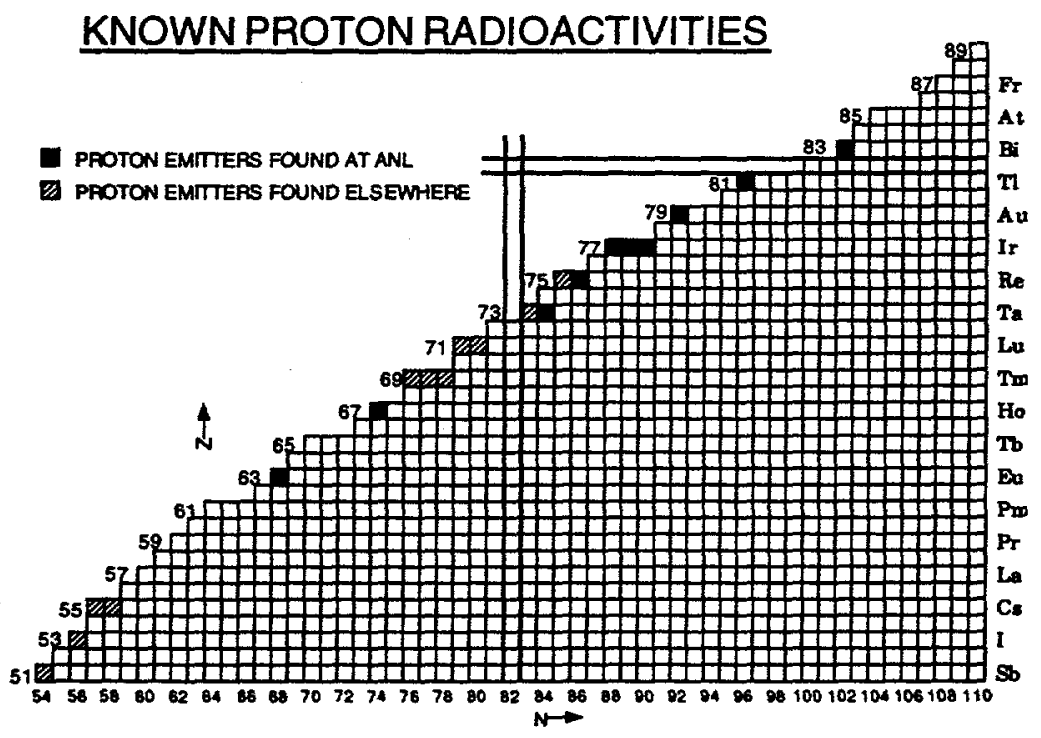

Figure 28: Known radioactive proton emitters ${ }^{36}$, which are found beyond the proton-drip line. They decay when a proton tunnels across its Coulomb barrier.

evaporation residues, very clean decay proton spectra can be observed, ${ }^{57}$ e.g. from ${ }^{167} \mathrm{Ir}$, as shown in Fig. 29(a). Proton decay is a simple but interesting case of tunneling of an elementary particle through its Coulomb barrier (Fig. 29(b). It is analogous to $\alpha$-decay, but is simpler to calculate since there is no need to evaluate a pre-formation factor. In general the proton tunneling rates can be calculated ${ }^{56,58}$ if the orbital angular momentum $\ell$ of the proton 

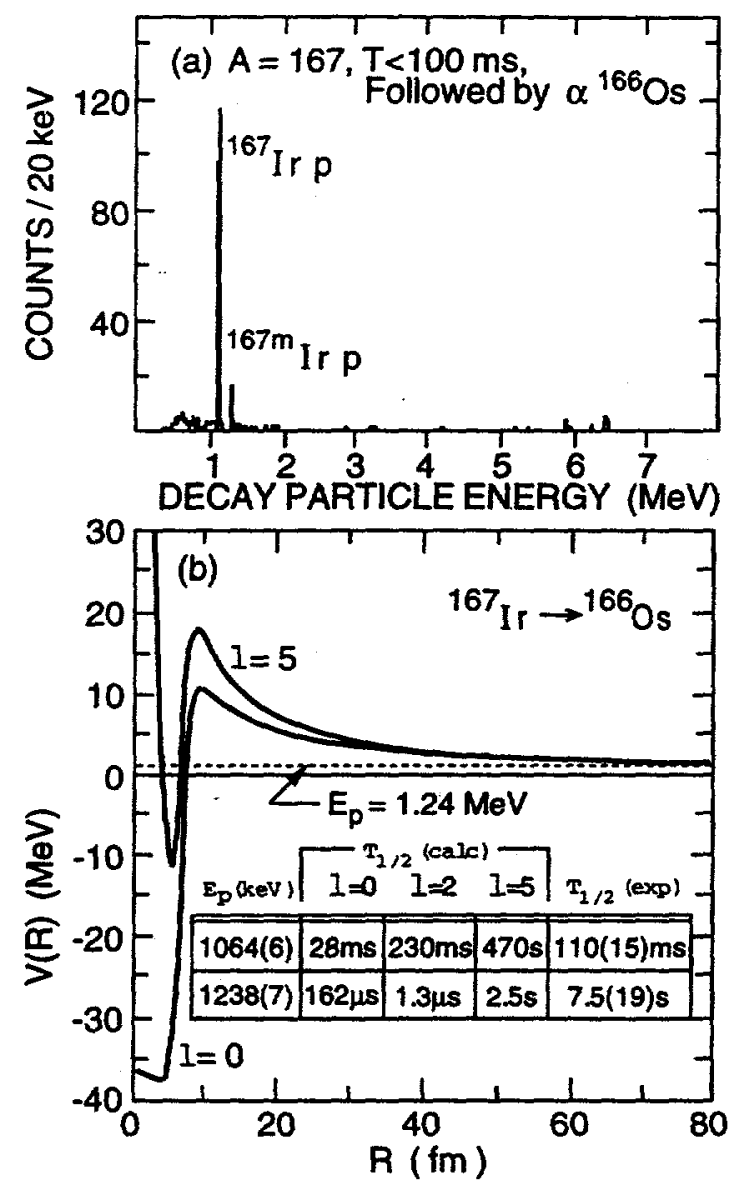

Figure 29: (a) Protons from the ground state and from an isomer in the proton emitter ${ }^{167} \mathrm{Ir}$ - from Ref. ${ }^{57}$. (b) Proton tunneling through Coulomb and centrifugal $(1=0,5)$ barriers from Ref. ${ }^{36}$.

is taken into account. Tunneling through a deformed potential is an interesting example of multi-dimensional tunneling, and examples of this phenomenon have recently been found ${ }^{59}$.

Tunneling from excited states of a proton emitter provides an opportunity to study cases where the initial state can change character from ordered to chaotic. One can also investigate the interplay between the proton energy and angular momentum: an excited high-spin state can take advantage of 


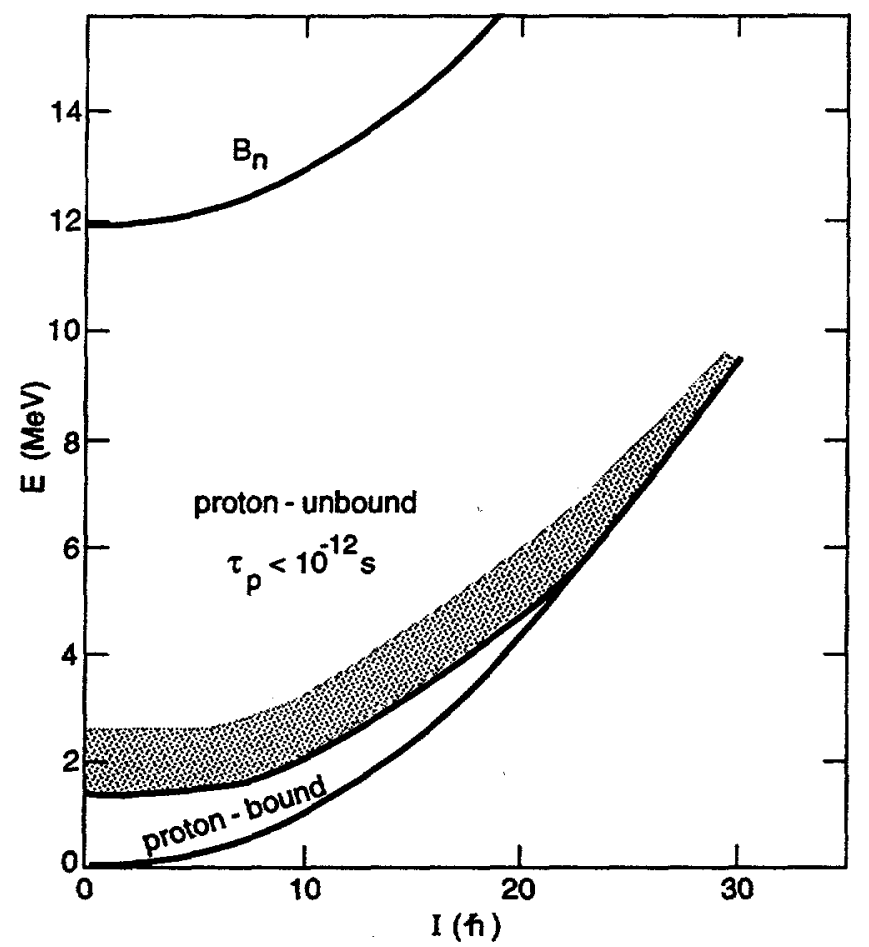

Figure 30: In a proton emitter, such as ${ }^{167} \mathrm{Ir}$, only a small spin-energy region is proton bound. The region above that, as well as the ground state, decay by proton tunneling. Protons from the dotted region are expected show a new phenomenon - sub-Coulomb peaks and structures. Levels above the line marked $B_{n}$ are neutron-unbound.

the increased energy for decay to lower-spin states to overcome the additional centrifugal barrier in decay with $\ell>0$.

Although the ground state decays by proton emission, states immediately above that, or along the yrast line, preferentially decay by $\gamma$ emission. However, $\Gamma_{p} \sim \Gamma_{\gamma}$ at some excitation energy, estimated to be $>1.2 \mathrm{MeV}$ above the yrast line in the proton emitter ${ }^{167} I r$; thereafter $\Gamma_{p}>\Gamma_{\gamma}$. As a consequence, it is expected that the proton-bound region of a proton-emitter is unusually small (Fig. 30). In contrast, in stable nuclei the particle-bound region is much larger, defined typically by the neutron separation energy of $\sim 8 \mathrm{MeV}$. Excited states in the dotted region of Fig. 30 should exhibit a new type of proton emission, namely sub-Coulomb protons that exhibit peaks and structures. It would be interesting to search for this new form of proton emission. As only the proton- 
bound region of Fig. 30 can lead to population of the proton-emitter ground state via $\gamma$ decay, there is another distinctive signature for the small bound region. The $\gamma$ ray spectrum, which normally extends to $\sim 8 \mathrm{MeV}$, will end at a much lower energy of $\sim 1.5 \mathrm{MeV}$ in a proton emitter. The prospective coupling of Gammasphere to the Fragment Mass Analyzer at Argonne will make it possible to examine the $\gamma$ spectra leading to the ground state of proton emitters.

\section{Comments on tunneling in nuclei}

The nucleus provides examples of tunneling where the initial and final states can be either ordered or disordered states. In the feeding of SD bands, tunneling between hot SD and ND states plays a role. In decay out of SD bands, an ordered state tunnels to a chaotic one. The tunneling probability is very small in the latter. However, it would have been even smaller if the tunneling were to another ordered state. In other words, the chaotic nature of the final state facilitates the tunneling rate ${ }^{60}$. This situation is related to chaos- assisted ${ }^{11}$ tunneling, where the transition between two ordered regions of phase space can be amplified by coupling with an intervening chaotic region. In a Poincaré surface of section, one visualizes a diffuse chaotic region surrounding the ordered regions and abetting the tunneling between them.

Restated in the conventional language of nuclear physics, one would say that the coupling between cold SD and ND states would be negligible because the states have different structures and quantum numbers. However, the compound nature of the hot ND state aids the coupling between SD and ND states, since compound states are able to couple to all states. One may also view this problem in terms of the shape parameters defined in Fig. 7. The SD state, well localized in $\beta$ and $\gamma$, can have some overlap with a hot ND state, which is spread out in the $\beta-\gamma$ plane. On the other hand, the overlap with a cold state, localized elsewhere in the plane, is considerably smaller and is also further reduced by a barrier.

For nuclei which lie beyond the proton drip line, proton decay from radioactive ground states and isomers connect cold initial and final nuclear states (although the proton is in the continuum representing an unbound final state). However, the character of the initial state can be changed from ordered to chaotic by increasing its excitation energy. This provides another incentive for studying proton emission from excited states of a proton emitter. 


\subsection{Analogies with other systems}

Tunneling from a SD state to the ND well can occur only when there is a nearby excited ND state. In other words, this is a resonant tunneling process. Some interesting analogies can be made with other processes where resonances are responsible for large-amplitude motion. In neutron- induced fission, intermediate resonances are observed -see Fig. 7 in Ref. ${ }^{3}$ - which represent enhanced fission when the neutron energy corresponds to a vibrational state in the secondary SD well of actinide nuclei. Molecular pre-dissociation, a process equivalent to nuclear fission, occurs when a bound level crosses an unbound one at some radial separation ${ }^{61}$. A third example comes from theoretical work 62 on a hydrogen atom in a polarized microwave field. A stable non-spreading electronic wave packet (Floquet state) is predicted. The crossing of the energy level of the wave packet with that of chaotic states (as a function of, say, the electric field strength) induces ionization of the stable wave packet, and spikes in the photoabsorption spectrum are predicted at these crossings.

\section{Summary}

The feeding and decay of SD bands are summarized in Fig. 31. Following neutron evaporation, there is an initial cooling by statistical $\gamma$ rays (component 1). This is the hot chaotic phase, where the nuclear shape is not well-defined because of thermal fluctuations. When the nucleus is well within the SD false vacuum, shape fluctuations begin to diminish, the elongated shape becomes established and collective E2 transitions from excited SD bands form a large E2 peak (component 2). Cooling into the SD minimum is brought about by M1/E2 transitions (component 3). Once trapped into the bottom of the SD vacuum, the nucleus undergoes cold, ordered rotation. The resultant spectrum, with characteristic equally-spaced sharp lines (component 4), is distinctly different from the broad, unresolved, precursor spectrum. A sudden tunneling to ND states signals a return to chaos, with a re-emergence of a broad statistical spectrum (component 5) and fluctuating primary-line strengths. Finally, the nucleus settles towards the ground state along the cold ND yrast line (component 6) by emission of sharp lines. Hence, SD nuclei provide examples of order embedded in chaos, display a unique double cycle of chaos-to-order transition, and exhibit tunneling from ordered to chaotic states, as well as between disordered SD and ND states. The decay spectra from SD bands reveal that the decay mechanism is a tunneling from an ordered state in the false vacuum to a chaotic one in the true vacuum. The high-energy, one-step decay transitions yield excitation energies and spin/parity quantum numbers, which characterize the SD bands as ordered states; reveal their microscopic structure; and sug- 
Feeding \& Decay of Superdeformed Bands in Hg Nuclei

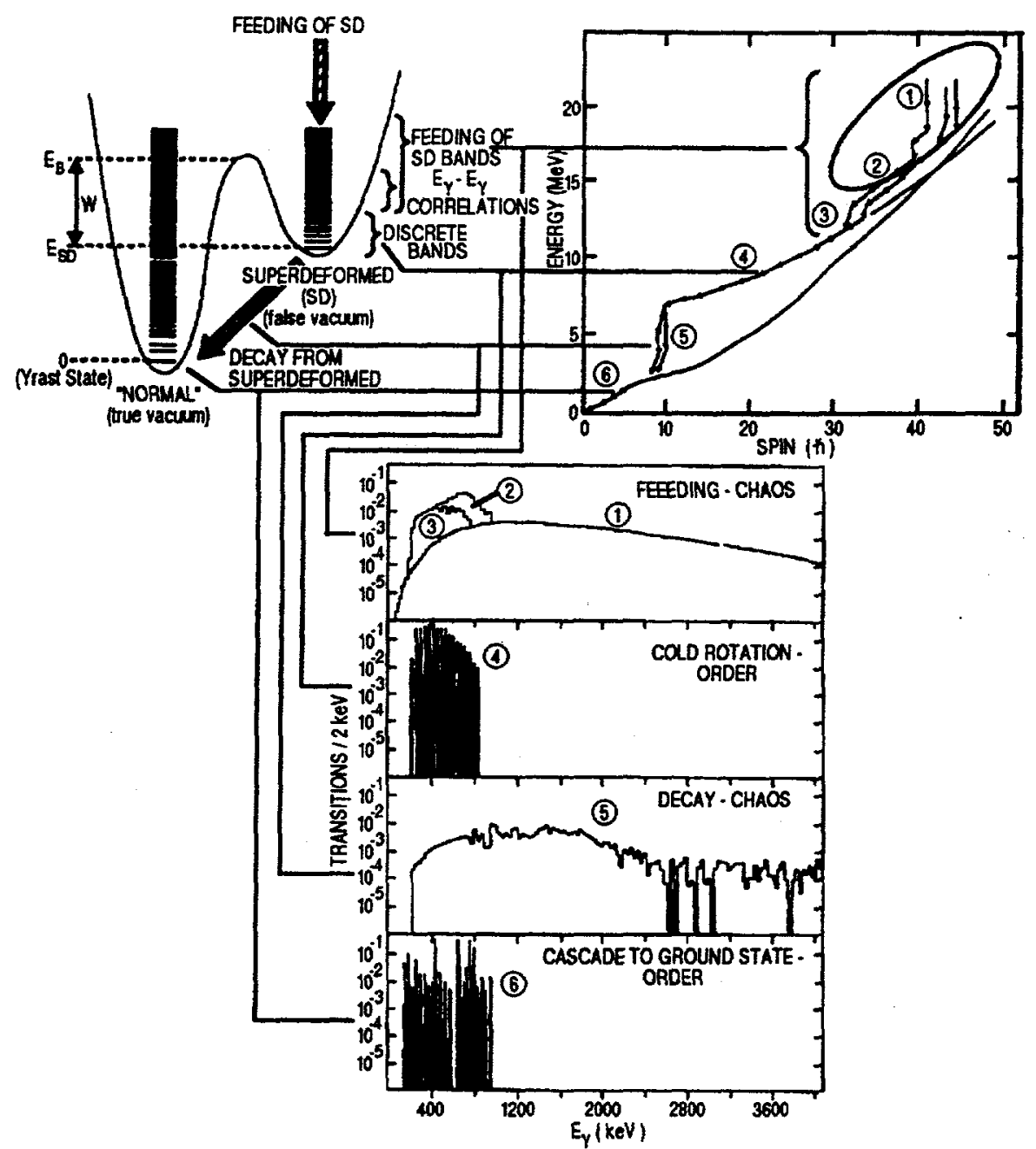

Figure 31: Gamma spectra from different stages in the feeding and decay of a SD band. The different characters of the spectra reveal a unique cycle of chaos-to-order-to-chaos-to-order transition. 
gest that identical bands arise from accidental cancellations between pairing and particle alignment. The decay spectrum also provides information about the quenching of pairing with excitation energy and indicates that excited ND states at $\sim 4 \mathrm{MeV}$ are largely chaotic.

\section{Acknowledgements}

A substantial portion of the work that I have described here is drawn from my own research. This work has only been possible because of the invaluable contributions and stimulation of my collaborators. It is with thanks and pleasure that I acknowledge T. Lauritsen, M. Carpenter, T. Døssing , G. Hackman, R. G. Henry, A. Lopez-Martens, D. Ackermann, I. Ahmad, H. Amro, D. Blumenthal, I. Calderin, D. Gassmann, R. Janssens, S. Fischer, F. Hannachi, A. Korichi, I. Y. Lee, A. Macchiavelli, F. Moore, T. Nakatsukasa, D. Nisius and P. Reiter. I wish to thank: C. Davids, R. Firestone and D. Haslip for providing some figures; R. Janssens for carefully reading this manuscript; and the Institute of Nuclear Theory for its hospitality during the program "Tunneling in Complex Systems", where these lectures were delivered. The work at Argonne National Laboratory is supported by the U. S. Department of Energy, under Contract No. W-31-109-ENG-38.

\section{References}

1. V. M. Strutinsky, Nucl. Phys. A 95, 420 (1967).

2. S. Hofmann, V. Ninov, F.P. Hessberger, P. Armbruster, H. Folger, G. Mnzenberg, H.J. Schtt, A.G. Popeko, A.V. Yeremin, S. Saro, R. Janik, M. Leino, Z. Phys. A 354, 229 (1996).

3. S. Bjørnholm and J. E. Lynn, Rev. Mod. Phys. 52, 725 (1980).

4. P. J. Nolan and P. J. Twin, Annu. Rev. Nucl. Part. Sci. 38, 533(1988).

5. R. V. F. Janssens and T. L. Khoo, Annu. Rev. Nucl. Part. Sci. 41, 321 (1991).

6. R. Krücken, G. Hackman, M. A. Deleplanque, R. V. F. Janssens, I. Y. Lee, D. Ackermann, I. Ahmad, H. Amro, S. Asztalos, D. J. Blumenthal, M. P. Carpenter, R. M. Clark, R. M. Diamond, P. Fallon, S. M. Fischer, B. Herskind, T. L. Khoo, T. Lauritsen, A. O. Macchiavelli, R. W. MacLeod, D. Nisius, G. Schmid, F. S. Stephens and K. Vetter, Phys. Rev. C 54, R2109 (1996).

7. A. Bohr and B. Mottelson, Nuclear Structure, vol. 2, p. 592 (W. A. Benjamin, Inc., Reading, 1975).

8. B. Singh, R. B. Firestone and S. Y. F. Chu, Table of Superdeformed Nuclear Bands and Fission Isomers, WWW Edition (1997); 
http://csa5.lbl.gov/ fchu/sdband/sdbindex.html.

9. R. U. Haq, A. Pandey and O. Bohigas, Phys. Rev. Lett. 481086 (1982).

10. B. Mottelson in The Frontier of Nuclear Spectroscopy, p. 7, ed. Y. Yoshizawa, H. Kusakari and T. Otsuka (World Scientific, Singapore, 1993).

11. O. Bohigas, D. Boose, R. Egydio de Carvalho and V. Marvulle, Nucl. Phys. A560, 197 (1993); S. Tomsovic and D. Ullmo, Phys. Rev. E 50, 145 (1994).

12. I. Y. Lee, Nucl. Phys. A520, 641c (1990).

13. T. Lauritsen, Ph. Benet, T. L. Khoo, K. B. Beard, I. Ahmad, M. P. Carpenter, P. J. Daly, M. W. Drigert, U. Garg, P. B. Fernandez, R. V. F. Janssens, E. F. Moore, F. L. H. Wolfs and D. Ye, Phys. Rev. Lett. 69,2479 (1992).

14. K. Schiffer and B. Herskind, Nucl. Phys. A520, 521c (1990).

15. T. L. Khoo, M. P. Carpenter, T. Lauritsen, D. Ackermann, I. Ahmad, D. Blumenthal, S. Fischer, R. V. F. Janssens, D. Nisius, E. F. Moore, A. Lopez-Martens, T. Døssing, R. Krücken, S. J. Asztalos, J. A. Becker, L. Bernstein, R. M. Clark, M. A.Deleplanque, R. M. Diamond, P. Fallon, L. P. Farris, F. Hannachi, E. A. Henry, A. Korichi, I. Y. Lee, A. O. Macchiavelli and F. S. Stephens, Phys. Rev. Lett. 76, 1583 (1996).

16. B. Herskind, T. Døssing, S. Leoni, M. Matsuo, N. Nica, D. C. Radford and P. Rasmussen, Nucl. Phys. A557, 191c (1993).

17. S. Leoni, B. Herskind, T. Døssing, A. Atac, I. G. Bearden, M. Bergstrom, C. Fahlander, G. B. Hagemann, A. Holm, D.T. Joss, M. Lipoglavsek, A. Maj, P. J. Nolan, J. Nyberg, M. Palacz, E. S. Paul, J. Persson, M. J. Piiparinen, N. Redon, A. T. Semple, G. Sletten and J.P. Vivien, Phys. Lett. B 409, 71 (1997).

18. E. F. Moore, T. Lauritsen, R. V. F. Janssens, T. L. Khoo, D. Ackermann, I. Ahmad, H. Amro, D. Blumenthal, M. P. Carpenter, S. M. Fischer, G. Hackman, D. Nisius, F. Hannachi, A. Lopez-Martens, A. Korichi, S. J. Asztalos, R. M. Clark, M. A.Deleplanque, R. M. Diamond, P. Fallon, I. Y. Lee, A. O. Macchiavelli F. S. Stephens, J. A. Becker, L. Bernstein, L. P. Farris and E. A. Henry, Phys. Rev. C 55, R2150 (1997).

19. C. Baktash, B. Haas and W. Nazarewicz, Annu. Rev. Nucl. Part. Sci. 45, 485 (1995).

20. E. Vigezzi, R. A. Broglia and T. Døssing, Phys. Lett. B 249, 163 (1990).

21. Y. R. Shimizu, E. Vigezzi, T. Døssing and R. A. Broglia, Nucl. Phys. A $557,99 \mathrm{c}(1993)$.

22. F. Barranco, G. F. Bertsch, R. A. Broglia and E. Vigezzi, Nucl. Phys. A512, 253 (1990). 
23. T. L. Khoo, T. Lauritsen, I. Ahmad, M. P. Carpenter, P. B. Fernandez, R. V. F. Janssens, E. F. Moore, F. L. H. Wolfs, Ph. Benet, P. J. Daly, K. B. Beard, U. Garg, D. Ye and M. W. Drigert, Nucl. Phys. A 557, 83c (1993).

24. F. Soramel, T. L. Khoo et al, to be published.

25. R. G. Henry, T. Lauritsen, T. L. Khoo, I. Ahmad, M. P. Carpenter, B. Crowell, T. Døssing , R. V. F. Janssens, F. Hannachi, A. Korichi, C. Schück, F. Azaiez, C. W. Beausang, R. Beraud, C. Bourgeois, R. M. Clark, I. Deloncle, J. Duprat, B. Gall, H. Hübel, M. J. Joyce, M. Kaci, Y. Lecoz, M. Meyer, E. S. Paul, N. Perrin, N. Poffe, M. G. Poquet, N. Redon, H. Sergolle, J. F. Sharpey-Schafer, J. Simpson, A. G.Smith, R. Wadsworth and P. Willsau, Phys. Rev. Lett. 73, 777 (1994).

26. B. Crowell, M. P. Carpenter, R. G. Henry, R. V. F. Janssens, T. L. Khoo, T. Lauritsen and D. Nisius, Nucl. Inst. Meth. A 355, 575 (1995).

27. D. C. Radford, I. Ahmad, R. Holzmann, R. V. F. Janssens and T. L. Khoo, Nucl. Inst. Meth. A 258, 111 (1987).

28. R. Holzmann, I. Ahmad, R. V. F. Janssens, T. L. Khoo and D. C. Radford, Nucl. Inst. Meth. A260, 153 (1987).

29. A. Lopez-Martens, F. Hannachi, T. Døssing , C. Schück, R. Collatz, E. Gueorguieva, Ch. Vieu, S. Leoni, B. Herskind, T. L. Khoo, T. Lauritsen, I. Ahmad, D. J. Blumenthal, M. P. Carpenter, D. Gassmann, R. V. F. Janssens, D. Nisius, A. Korichi, C. Bourgeois, A. Astier, L. Ducroux, Y. Le Coz, M. Meyer, N. Redon, J. F. Sharpey-Schafer, A. N. Wilson, W. Korten, A. Bracco and R. Lucas, Phys. Rev. Lett. 77, 1707 (1996).

30. T. Lauritsen, A. Lopez-Martens, T. L. Khoo, R. V. F. Janssens, M. P. Carpenter, G. Hackman, D. Ackermann, I. Ahmad, H. Amro, D. J. Blumenthal, S. M. Fischer, F. Hannachi, A. Korichi and D. Nisius in Proc. Conf. On Nuclear Structure at the Limits, Argonne, 1996, ANL/PHY$97 / 1$, p. 35.

31. G. Hackman, T. L. Khoo, M. P. Carpenter, T. Lauritsen, A. LopezMartens, I.V. Calderin, R. V. F. Janssens, D. Ackermann, I. Ahmad, S. Agarwala, D. J. Blumenthal, S. M. Fischer, D. Nisius, P. Reiter, J. Young, H. Amro, E. F. Moore, F. Hannachi, A. Korichi, I. Y. Lee, A. O. Macchiavelli, T. Døssing and T. Nakatsukasa, Phys. Rev. Lett. 79, 4100 (1997).

32. A. Lopez-Martens, F. Hannachi, A. Korichi, C. Schück, E. Gueorguieva, Ch. Vieu, B. Haas, R. Lucas, A. Astier, G. Baldsiefen, M. Carpenter, G. de France, R. Duffait, L. Ducroux, Y. Le Coz, Ch. Fink, A. Gorgen, H. Hübel, T. L. Khoo, T. Lauritsen, M. Meyer, D. Pevost, N. Redon, C. Rigollet, H. Savajols, J. F. Sharpey-Schafer, O. Stezowki, Ch. Theisen, 
U. Van Severen, J.P. Vivien and A. N. Wilson, Phys. Lett. B 380, 18 (1996).

33. K. Hauschild, L. A. Bernstein, J. A. Becker, D. E. Archer, R. W. Bauer, D. P. McNabb, J. A. Cizewski, K.-Y. Ding, W. Younes, R. Krücken, R. M. Diamond, R. M. Clark, P. Fallon, I.-Y. Lee, A. O. Macchiavelli, R. MacLeod, G. J. Schmid, M. A. Deleplanque, F. S. Stephens and W. H. Kelly, Phys. Rev. C 55, 2819 (1997).

34. J. Schirmer, J. Gerl, D. Habs and D. Schwalm, Phys. Rev. Lett. 63, 2196 (1989).

35. S. Lunardi, R. Venturelli, D. Bazzaco, C. M. Petrache, C. Rossi- Alvarez, G. de Angelis, G. Vedovato, D. Bucurescu and C. Ur, Phys. Rev. C 52, R6 (1995), and references therein.

36. C. E. Porter and R. G. Thomas, Phys. Rev. 104, 483 (1956).

37. T. Nakatsukasa, K. Matsuyanagi, S. Mizutori and Y. R. Shimizu, Phys. Rev. C 53, 2213 (1996).

38. B. Crowell, R. V. F. Janssens, M. P. Carpenter, I. Ahmad, S. Harfenist, R. G. Henry, T. L. Khoo, T. Lauritsen, D. Nisius, A. N. Wilson, J. Sharpey- Schafer and J. Skalski, Phys. Lett. B 333, 320 (1994).

39. S. Bouneau, F. Azaiez, J. Duprat, I. Deloncle, M-G. Porquet, U. J. van Severen, T. Nakatsukasa, M. M. Aleonard, A. Astier, S. Baldsiefen, C. W. Beausang, F. A. Beck, C. Bourgeois, D. Curien, N. Dozie, L. Ducroix, B. Gall, H. Hübel, M. Kaci, W. Korten, M. Meyer, N. Redon, H. Sergolle, and J. F. Sharpey-Schafer, in Proc. Conf. On Nuclear Structure at the Limits, Argonne, 1996, ANL/PHY-97/1, p. 105.

40. P. H. Heenen, J. Dobaczewski, W. Nazarewicz, P. Bonche and T. L. Khoo, preprint (1997).

41. J. E. Draper, F. S. Stephens, M. A. Deleplanque, W. Korten, R. M. Diamond, W. H. Kelly, F. Azaiez, A. O. Macchiavelli, C. W. Beausang and E. C. Rubel, Phys. Rev. C 42, R1791 (1990); J. Becker, E. A. Henry, A. Kuhnert, T. F. Wang, S. W. Yates, R. M. Diamond, F. S. Stephens, J. E. Draper, W. Korten, M. A. Deleplanque, A. O. Macchiavelli, F. Azaiez, W. H. Kelly, J. A. Cizewski and M. J. Brinkman, ibid. C 46, 889 (1992).

42. S. M. Harris, Phys. Rev. 138, 508B (1965).

43. P. Fallon, W. Nazarewicz, M. Riley and R. Wyss, Phys. Lett. B 276, 427 (1992).

44. R. Wyss and W. Satuła, Phys. Lett. B 351, 393 (1995).

45. T. Døssing , T. L. Khoo, T. Lauritsen, I. Ahmad, D. Blumenthal, M. P. Carpenter, B. Crowell, D. Gassmann, R. G. Henry, R. V. F. Janssens and D. Nisius, Phys. Rev. Lett. 75, 1276 (1995).

46. S. S. M. Wong, Nuclear Statistical Spectroscopy, p. 32 (Oxford Univ. 
Press, Oxford 1986).

47. H. E. Jackson, J. Julien, S. Samour, A. Bloch, C. Lopata, J. Morgenstern, H. Mann and G. E. Thomas, Phys. Rev. Lett. 17,656 (1966).

48. A. Lopez-Martens et al, to be published.

49. S. Flibotte, H. R. Andrews, G. C. Ball, C. W. Beausang, F. A. Beck, G. Belier, T. Byrski, D. Curien, P. J. Dagnall, G. de France, D. Disdier, G. Duchene, Ch. Finck, B. Haas, G. Hackman, D. S. Haslip, V. P. Janzen, B. Kharraja, J. C. Lisle, J. C. Merdinger, S. M. Mullins, W. Nazarewicz, D. C. Radford, V. Rauch, H. Savajols, J. Styczen, Ch. Theisen, P.J. Twin, J. P. Vivien, J. C. Waddington, D. Ward, K. Zuber and S. Åberg, Phys. Rev. Lett. 71, 4299 (1993).

50. D. S. Haslip, S. Flibotte, G. de France, M. Devlin, A. Galindo- Uribarri, G. Gervais, G. Hackman, D. R. LaFosse, I. Y. Lee, F. Lerma, A. O. Macchiavelli, R. W. MacLeod, S. M. Mullins, J. M. Nieminen, D. G. Sarantites, C. E. Svensson, J. C. Waddington and J. N. Wilson, Phys. Rev. Lett. 78, 3447 (1997).

51. I. Hamamoto and B. Mottelson, Phys. Lett. B 333, 294 (1994); Phys. Scr. T 56, 27 (1995).

52. S. Hofmann, V. Ninov, F.P. Hessberger, P. Armbruster, H. Folger, G. Mnzenberg, H.J. Schtt, A.G. Popeko, A.V. Yeremin, A.N. Andreyev, S. Saro, R. Janik, M. Leino, Z. Phys. A 350, 277 (1995)

53. R. Vandenbosch, Annu. Rev. Nucl. Part. Sci. 42, 447 (1992).

54. A. Winter, Nucl. Phys. A594, 203 (1995).

55. S. Hofmann in Nuclear Decay Modes p.143, ed. D. N. Poenaru, (Institute of Physics Publishing, 1996).

56. P. J. Woods and C. N. Davids, Annu. Rev. Nucl. Part. Sci. 47, 541 (1997).

57. C. N. Davids, P. J. Woods, J. C. Batchelder, C. R. Bingham, D. J. Blumenthal, L. T. Brown, B. C. Busse, L. F. Conticchio, T. Davinson, S. J. Freeman, D. J. Henderson, R. J. Irvine, R. D. Page, H. T. Penttila, D. Seweryniak, K. S. Toth, W. B. Walters and B. E. Zimmerman, Phys. Rev. C 55, 2255 (1997).

58. S. Áberg, P. B. Semmes and W. Nazarewicz, Phys. Rev. C56, 1762 (1997).

59. C. N. Davids, priv. com. (1997).

60. O. Bohigas, priv. com. (1997).

61. L. D. Landau and E. M. Lifshitz, Quantum Mechanics, Non-Relativistic Theory, p. 310 (Pergamon Press, London, 1958).

62. Z. Zakrewski, D. Delande and A. Buchleitner, Phys. Rev. Lett. 75, 4105 (1995). 